\title{
A scalar imaging velocimetry technique for fully resolved four-dimensional vector velocity field measurements in turbulent flows
}

\author{
Werner J. A. Dahm, Lester K. Su, and Kenneth B. Southerland \\ Department of Aerospace Engineering, The University of Michigan, Ann Arbor, Michigan 48109-2140
}

(Received 27 December 1991; accepted 10 June 1992)

\begin{abstract}
This paper presents an experimental technique for obtaining fully resolved measurements of the vector velocity field $\mathbf{u}(\mathbf{x}, t)$ throughout a four-dimensional spatiotemporal region in a turbulent flow. The method uses fully resolved four-dimensional scalar field imaging measurements in turbulent flows [Phys. Fluids A 3, 1115 (1991)] to extract the underlying velocity field from the exact conserved scalar transport equation. A procedure for accomplishing this is described, and results from a series of test cases are presented. These involve synthetically generated scalar fields as well as actual measured turbulent flow scalar fields advected numerically by various imposed flow fields. The imposed velocity fields are exactly known, allowing a careful validation of the tcchnique and its potential accuracy. Results obtained from a zeroth iteration of the technique are found to be very close to the exact underlying vector velocity field. Further results show that successive iterations bring the velocity field from the zeroth iteration even closer to the exact result. It is also shown that the comparatively dense velocity field information that this technique provides is well suited for accurate extraction of the more dynamically insightful strain rate and vorticity fields $\epsilon(\mathbf{x}, t)$ and $\omega(\mathbf{x}, t)$.
\end{abstract}

\section{INTRODUCTION}

The inability to measure fully resolved fourdimensional space- and time-varying vector velocity fields $\mathbf{u}(\mathbf{x}, t)$ in complex flows presents one of the biggest obstacles in the study of the physics of fluid flows. The need for such an experimental technique is arguably greatest in turbulent flows, where relatively little is known about the detailed spatial structure and dynamics of the vector vorticity fields and tensor strain rate fields on the inner scales of such flows. Because of the lack of such a suitable measurement technique, investigations of the fine scale structure and dynamics in turbulent flows have been essentially restricted to large-scale direct numerical simulations (DNS) of the full equations of fluid motion. While such simulations have indeed provided considerable insight, they remain limited to comparatively simple flows such as homogeneous, isotropic or sheared turbulence at relatively low Reynolds numbers. The fine structure and dynamics of the velocity field-and, in particular, of the more dynamically insightful vorticity and strain rate fields-in real, inhomogeneous, anisotropic turbulent shear flows at even moderately high outer-scale Reynolds numbers presently remain inaccessible to direct study.

There are many cxpcrimental techniques currently in use for velocity measurements in turbulent flows. Most of these are inherently single-point techniques, producing a time series of one or more components of the velocity vector at a single point in space. These include the whole class of invasive probe techniques, the most commonly used being hot-wire and hot-film anemometry. More contemporary noninvasive optical techniques also exist, of which laser Doppler velocimetry is by far the most popular. Limited spatial or temporal coherence information can be extracted at relatively coarse scales of the flow by using such single-point techniques simultaneously at a number of points to produce a spatial array of time series data. ${ }^{1-8}$ However, the low spatial resolution attainable by such multiple single-point measurements renders them unsuitable for evaluating the local spatial derivatives necessary to extract the underlying fine scale structure and dynamics of turbulent flows.

Recently developed two-dimensional optical imaging methods permit measurements of vector velocities simultaneously at a large number of points in a flow. Reviews of some of these techniques are given by Adrian, ${ }^{9,10}$ Merzkirch, ${ }^{11}$ Lauterborn and Vogel, ${ }^{12}$ Hesselink, ${ }^{13}$ Miles and Nosenchuck, ${ }^{14}$ Chang and Reid, ${ }^{15}$ and Gad-el-Hak. ${ }^{16}$ The majority of these techniques involve particle imaging methods, among them laser speckle velocimetry, ${ }^{17-24}$ particle tracking velocimetry, ${ }^{25-29}$ and particle image velocimetry. ${ }^{30-33}$ These typically yield the projection of the velocity vectors in a single measurement plane. Threedimensional particle tracking ${ }^{34,35}$ and holographic particle image velocimetry ${ }^{36-38}$ are now being explored as techniques for measuring full three-component vector velocity fields in complex flows. However, even with these multipoint measurement techniques, accurate spatial differentiation to extract the underlying fine structure of the vorticity and strain rate fields remains compromised by the comparatively low spatial and temporal resolution attainable. Various laser-induced fluorescence and phosphorescence techniques $^{39}$ have also been considered, some of which allow direct extraction of the velocity gradient field, ${ }^{40}$ but these too appear unsuitable for studies of the fine scale dynamics of turbulent flows at Reynolds numbers of interest.

Here, we take an entirely different approach to the measurement of the fully resolved, four-dimensional, vector velocity field $\mathbf{u}(\mathbf{x}, t)$ on the inner scales of turbulent 
shear flows. Our approach is rooted in the recently demonstrated experimental capability for obtaining fully resolved four-dimensional measurements of the fine scale structure of $\mathrm{Sc} \gg 1$ conserved scalar fields $\zeta(\mathbf{x}, t)$ on the inner scales of turbulent flows. ${ }^{41-43}$ In this paper, we present a technique for extracting the underlying spaceand time-varying vector velocity field from such scalar field measurements of a single, dynamically passive conserved scalar in a turbulent flow. The technique involves inverting the exact conserved scalar transport equation throughout the dense, four-dimensional, spatiotemporal data space to directly yield the velocity component field $u_{\|}(\mathbf{x}, t)$ along the local scalar gradient vector $\nabla \xi(x, t)$. An examination of the corresponding gradient field $\nabla u_{\|}(\mathrm{x}, t)$ then allows extraction of the full vector velocity field $\mathbf{u}(\mathbf{x}, t)$. We describe this approach in detail and apply it to a series of test cases in which synthetically generated scalar fields as well as actual measured turbulent flow scalar fields are numerically advected by various imposed flow fields. Since the objective velocity fields are exactly known, we are able to obtain a careful validation of this scalar imaging velocimetry technique as well as an indication of its potential accuracy.

The presentation is organized as follows. In Sec. II, we describe the scalar imaging velocimetry technique. Section III presents results from several test cases that allow a validation of the method and an assessment of its capabilities, and in Sec. IV we discuss the general utility of this method and draw conclusions regarding its suitability for permitting direct experimental studies of the fine scale structure and dynamics of the vector velocity fields, as well as the vorticity vector field and the strain rate tensor field, on the inner scale of turbulent flows. The Appendix describes the numerical techniques used here to implement this technique in the test cases presented.

\section{SCALAR IMAGING VELOCIMETRY}

In this section, we describe the approach used to extract the vector velocity field $\mathbf{u}(\mathbf{x}, t)$ from fully resolved four-dimensional measurements of a single, dynamically passive, conserved scalar field $\zeta(\mathbf{x}, t)$ in turbulent flows. We begin with a brief overview of the experimental method currently in use for measuring scalar field data of the type to which the present velocimetry technique is to be applied. Details of such turbulent scalar field measurements, as well as examples of the data obtained, are presented in Refs. 42 and 43 . Here, we only summarize those aspects that are essential to the extraction of the underlying velocity field.

Briefly, such fully resolved four-dimensional scalar field measurements are based on successive, high-speed, planar imaging of the laser-induced fluorescence from a dynamically passive laser dye carried by the flow, whose concentration is a conserved scalar variable. A collimated laser beam is repeatedly swept in a raster fashion throughout a small volume in the flow by a pair of low-inertia mirrors driven by two galvanometric scanners slaved to the imaging array timing. The successive $256 \times 256$ scalar field data planes are acquired at rates up to 142 planes/sec into gigabyte-sized data sets using very fast computer disk ranks to produce four-dimensional spatiotemporal data space structures as shown in Fig. 2 of Ref. 43. Each such measured data space consists of a rapid succession of individual three-dimensional spatial data volumes. Each of these data volumes in turn consists of a sequence of twodimensional spatial data planes, each of which consists of an array of $256 \times 256$ individual data points. The flow facility and imaging electronics are designed so that the resulting effective spatial resolution $(\Delta x, \Delta y, \Delta z)$ between adjacent points in each three-dimensional data volume is smaller than the local strain-limited molecular diffusion scale $\lambda_{D} / \delta \sim \mathrm{Sc}^{-1 / 2} \mathrm{Re}_{\delta}^{-3 / 4}$ of the scalar field. Similarly, the temporal resolution $\Delta t$ between the same data point in successive spatial data volumes is smaller than the local molecular diffusion scale advection time $\lambda_{D} / u$. This resolution, together with the high signal quality attained, allows accurate differentiation of the measured conserved scalar field data in all three space dimensions and in time to determine the components of the instantaneous time derivative field $(\partial / \partial t) \xi(\mathbf{x}, t)$, the scalar gradient vector field $\nabla \zeta(\mathbf{x}, t)$, and the Laplacian field $\nabla^{2} \zeta(\mathbf{x}, t)$ throughout the four-dimensional data space. Such measurements have been reported at outer-scale Reynolds numbers $\operatorname{Re}_{\delta} \equiv(u \delta / v)$ as high as 6000 with individual fully resolved data planes spanning an area as large $1.6 \lambda_{v} \times 1.6 \lambda_{v}$.

Of practical relevance for the present discussion, note that this technique produces the measured scalar field $\zeta(\mathbf{x}, t)$ at every point on a dense and regular grid in the four-dimensional spatiotemporal data space. Moreover, the scalar diffusivity is typically quite small, or more precisely the Schmidt number is large. For example, in the disodium fluorescein measurements referred to above, $\mathrm{Sc} \approx 2075$. As a consequence, the underlying velocity field that we are aiming here to extract from such measurements is considerably smoother than the scalar field from which we begin. In particular, the finest gradient length scale in $\lambda_{v}$ in the velocity field is larger, by a factor of $\mathrm{Sc}^{1 / 2}$, than the smallest gradient length scale $\lambda_{D}$ in the scalar field, so this ratio of scales is about 45 for the measurements referred to above.

\section{A. Direct extraction of $u_{\|}(x, t)$ from $\zeta(x, t)$}

Any such dynamically passive, conserved scalar field $\zeta(\mathbf{x}, t)$ follows the exact advective-diffusive transport equation

$$
\left(\frac{\partial}{\partial t}+\mathbf{u} \cdot \nabla-\frac{1}{\operatorname{ReSc}} \nabla^{2}\right) \zeta(\mathbf{x}, t)=0 .
$$

Here, and throughout, all variables are taken to be nondimensionalized with reference length and velocity scales, $l$ * and $u^{*}$, and reference scalar value $\zeta^{*}$. In Eq. (1), the dimensionless scalar diffusivity $(1 / \mathrm{Re} \mathrm{Sc})$ involves the product of the Reynolds number $\operatorname{Re} \equiv\left(u^{*} l * / v\right)$ and the Schmidt number $\mathrm{Sc} \equiv(v / D)$, where $v$ and $D$ are the vorticity and scalar diffusivities, respectively.

Note in Eq. (1) that the influence of the underlying fluid velocity field $\mathbf{u}(\mathbf{x}, t)$ on the scalar field $\zeta(\mathbf{x}, t)$ is strictly through the convective term $\mathbf{u} \cdot \nabla \zeta$. This can be written as $u_{\|}|\nabla \zeta|$, where $u_{\|}$represents the component of the 


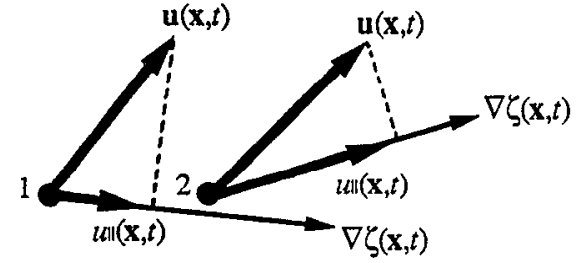

FIG. 1. Schematic showing the difference in $u_{\|}(\mathbf{x}, t)$ between two closely spaced points due to the change in $\mathbf{u}(\mathbf{x}, t)$ and the change in $\hat{e}_{\nabla 5}(\mathbf{x}, t)$.

local velocity vector along the local scalar gradient vector direction. Four-dimensional measurements of the scalar field $\zeta(x, t)$, with resolution and signal quality sufficient to allow accurate direct differentiation in both space and time, allow extraction of this velocity component throughout the spatiotemporal data space as

$$
u_{\|}(\mathbf{x}, t)=\left(\frac{1}{\operatorname{ReSc}} \nabla^{2} \xi(\mathbf{x}, t)-\frac{\partial \zeta(\mathbf{x}, t)}{\partial t}\right)[|\nabla \zeta(\mathbf{x}, t)|]^{-1} .
$$

This involves not only the scalar gradient field $\nabla \zeta(\mathbf{x}, t)$, but also the Laplacian field $\nabla^{2} \zeta(\mathbf{x}, t)$ and the time derivative field $(\partial / \partial t) \xi(\mathbf{x}, t)$. The inversion is, of course, only possible where the local scalar gradient vector magnitude is not zero.

Southerland et al. ${ }^{44}$ recently reported the measurement of $u_{\|}(\mathbf{x}, t)$ using Eq. (2) for the relatively simple flow field represented by an axisymmetric laminar vortex ring. Their results, which also show the individual component fields involved in Eq. (2), used linear central difference approximations, without any explicit smoothing or filtering, on the measured scalar field $\xi(\mathbf{x}, t)$ to numerically evaluate the scalar gradient magnitude field $|\nabla \xi(\mathbf{x}, t)|$ as well as the time derivative field $(\partial / \partial t) \zeta(\mathbf{x}, t)$ and the Laplacian field $\nabla^{2} \xi(\mathrm{x}, t)$. Figure 6 of Ref. 44 shows that remarkably clean first- and second-derivative fields are indeed obtainable from measured four-dimensional conserved scalar field data.

\section{B. Local variations in $u_{\|}(x, t)$}

From Eq. (2), the value of $u_{\|}(x, t)$ at any point in the spatiotemporal data space gives the projection of the local $\mathbf{u}(\mathbf{x}, t)$ onto the unit vector $\hat{e}_{\nabla \zeta}(\mathbf{x}, t)$ pointing in the local scalar gradient vector direction, namely

$$
u_{\|}(\mathbf{x}, t)=\mathbf{u}(\mathbf{x}, t) \cdot \hat{e}_{\nabla \zeta}(\mathbf{x}, t),
$$

where

$$
\hat{e}_{\nabla \zeta}(\mathbf{x}, t) \equiv \nabla \zeta(\mathrm{x}, t) /|\nabla \zeta(\mathbf{x}, t)| .
$$

As a result, $u_{\|}(\mathrm{x}, t)$ differs between any two points $\mathrm{x}$ and $\mathbf{x}+d \mathbf{x}$ due to (i) the change in the gradient vector direction, which is known, and (ii) the change in $\mathbf{u}(\mathbf{x}, t)$. This is shown schematically in Fig. 1, and can be expressed formally as

$$
\nabla u_{\|}=\mathbf{u} \cdot \nabla \hat{e}_{\nabla \zeta}^{T}+\nabla \mathbf{u}^{T} \cdot \hat{e}_{\nabla \zeta} .
$$

Note that the gradient fields are, for the present time at least, approximated linearly as

$$
\mathbf{u}(\mathbf{x}+d \mathbf{x}) \equiv \mathbf{u}(\mathbf{x})+\nabla \mathbf{u} \cdot d \mathbf{x}
$$

and

$$
\hat{e}_{\nabla \zeta}(\mathbf{x}+d \mathbf{x}) \equiv \hat{e}_{\nabla \zeta}(\mathbf{x})+\nabla \hat{e}_{\nabla \zeta} \cdot d \mathbf{x}
$$

In Eq. (4), the $u_{\|}(\mathbf{x}, t)$ field and the scalar gradient vector orientation field $\hat{e}_{\nabla \zeta}(\mathbf{x}, t)$ are known from the measured scalar field data. The unknowns are the three components of the velocity field $\mathbf{u}(\mathbf{x}, t)$ and the nine components of its gradient field $\nabla \mathbf{u}(\mathbf{x}, t)$. If $\mathrm{Eq}$. (4) were interpreted purely locally, then only three linearly independent equations could be formed from it and there would be little hope of extracting $\mathbf{u}(\mathbf{x}, t)$ and $\nabla \mathbf{u}(\mathbf{x}, t)$. Of course, there are, in fact, only three unknowns in Eq. (4), since the nine components of $\boldsymbol{\nabla u}(\mathbf{x}, t)$ result directly from relations among adjacent values of $\mathbf{u}(\mathbf{x}, t)$. The availability of such adjacency information from the three-dimensional spatial character of the original scalar field measurements motivates an iterative procedure to find the velocity field $\mathbf{u}(\mathbf{x}, t)$ in Eq. (4).

\section{The zeroth iteration for $u(x, t)$}

Recall from Fig. 1 that variations in $u_{\|}(\mathbf{x}, t)$ between any two points $\mathrm{x}$ and $\mathrm{x}+d \mathrm{x}$ are due to variations in both $\mathbf{u}(\mathbf{x}, t)$ and $\hat{e}_{\nabla \zeta}(\mathbf{x}, t)$. We begin by examining the characteristic length scales over which $\mathbf{u}(\mathbf{x}, t)$ and $\hat{e}_{\mathbf{v} \xi}(\mathbf{x}, t)$ can be expected to vary. For large Sc scalar mixing in turbulent flows, we can expect that the scalar gradient field $\nabla \xi(\mathbf{x}, t)$ will contain considerably more fine structure than the velocity gradient field $\nabla \mathbf{u}(\mathbf{x}, t)$. In particular, at any point in the flow, the influence of a locally uniform but timevarying strain field $\epsilon(t)$ leads to a competition between the effects of strain and diffusion which establishes an equilibrium strain-limited vorticity diffusion length scale $\lambda_{v} \sim(v / \epsilon)^{1 / 2}$, closely related to the Kolmogorov scale, giving the finest scale on which spatial gradients in the vorticity field can be locally sustained. A similar competition leads to a local strain-limited scalar gradient lengthscale $\lambda_{D} \sim(D / \epsilon)^{1 / 2}$, related to the Batchelor scale. The velocity gradient field $\boldsymbol{\nabla u}(\mathbf{x}, t)$ will then be linear over lengthscales of the order of $\lambda_{v}$, while $\nabla \xi(\mathbf{x}, t)$ is linear over lengthscales of order $\lambda_{D} \approx \lambda_{v} \cdot \mathrm{Sc}^{-1 / 2}$, so that, for large Schmidt numbers, $\lambda_{D} \ll \lambda_{v}$

It must be noted, however, that it is principally the magnitude of $\nabla \zeta$ that varies over this length scale. The unit vector $\hat{e}_{\nabla \zeta}$ pointing along the local $\nabla \zeta(\mathbf{x}, t)$ direction will vary over somewhat larger length scales. This can be seen, for example, in Figs. 5 and 6 of Ref. 43. Indeed, there is evidence that, over most of the scalar field, $\nabla \xi(\mathbf{x}, t)$ tends to remain largely aligned with the most compressive local principal strain rate axis of the velocity gradient field, which, in turn, varies over length scales of the order of $\lambda_{v}$. Nevertheless, owing to the continual stretching and folding action of the scalar dissipation layers by the $\nabla \mathbf{u}(\mathbf{x}, t)$ field, there will be regions in the flow in which the local $\nabla \xi$ does not stay aligned with the principal strain rate axes. Such 


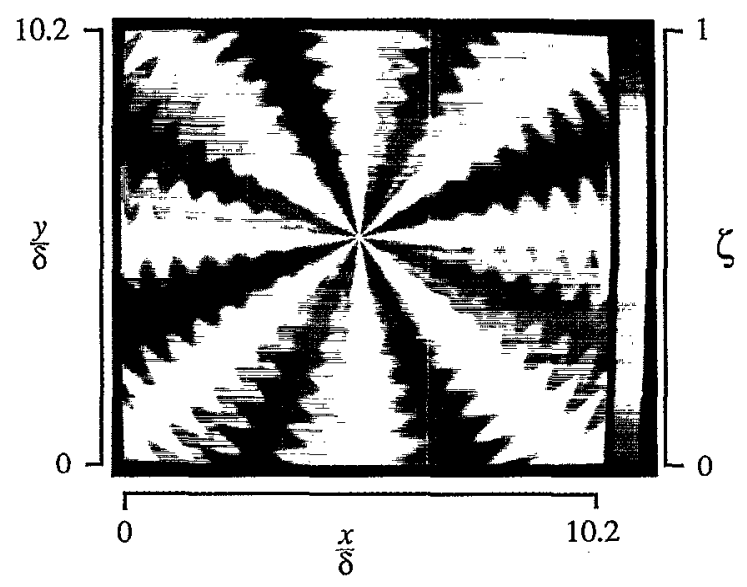

FIG. 2. The original synthetic conserved scalar field $\xi(\mathbf{x}, t)$ for Case $I$ in Sec. III A.

areas can be readily identified, for example, in Figs. 5 and 6 of Ref. 43, where the scalar gradient layers fold back onto themselves within a small region. In these regions at least, we can expect $\hat{e}_{\nabla \zeta}$ to vary with $d \mathbf{x}$ more rapidly than will $\mathbf{u}$.

We can therefore generate a starting solution for the velocity field in such regions by initially ignoring the contribution from variations in $\mathbf{u}(\mathbf{x}, t)$ to the spatial variations in $u_{\|}(\mathbf{x}, t)$. This amounts to assuming that, for small $d \mathbf{x}$, $\delta \mathbf{u} \cdot \hat{e}_{\nabla \zeta}$ in Eq. (4) is small in comparison with $\mathbf{u} \cdot \delta \hat{e}_{\mathbf{v}}$. In such a small neighborhood around any point $\mathbf{x}$, the $u_{\|}$'s found at three different points are then essentially the projections of a single local velocity vector $\boldsymbol{u}$ onto the three different unit vectors $\hat{e}_{\nabla \xi}$. For any three points $i=\{1,2,3\}$ we can express this formally by writing Eq. (3a) in terms of the local projection matrix as

$$
\left[\begin{array}{l}
\left(u_{\|}\right)_{1} \\
\left(u_{\|)_{2}}\right. \\
\left(u_{\|}\right)_{3}
\end{array}\right]=\left[\begin{array}{lll}
\left(\hat{e}_{\nabla \zeta}\right)_{1, x} & \left(\hat{e}_{\nabla \zeta}\right)_{1, y} & \left(\hat{e}_{\nabla \zeta}\right)_{1, z} \\
\left(\hat{e}_{\nabla \zeta}\right)_{2, x} & \left(\hat{e}_{\nabla \zeta}\right)_{2, y} & \left(\hat{e}_{\nabla \zeta}\right)_{2, z} \\
\left(\hat{e}_{\nabla \zeta}\right)_{3, x} & \left(\hat{e}_{\nabla \zeta}\right)_{3, y} & \left(\hat{e}_{\nabla \zeta}\right)_{3, z}
\end{array}\right]\left[\begin{array}{c}
u \\
v \\
w
\end{array}\right],
$$

where each of the matrix elements $\left(\hat{e}_{\nabla \zeta}\right)_{i, j}$ is the $x_{j}$ component of the unit vector $\hat{e}_{\nabla \zeta}$ at the $i$ th point. If the points are selected so that the three $\hat{e}_{\nabla \xi}$ 's are sufficiently noncolinear, then the local velocity vector $\mathbf{u}=(u, v, w)$ can be obtained from the measured $u_{\|}(\mathbf{x}, t)$ via the inverse of this local projection matrix. Of course, in any such small neighborhood, there are in principle many combinations of points for which Eq. (6) can be written and from which estimates for the local velocity vector $\mathbf{u}$ can be obtained. This high level of redundancy leads to a strong probability of finding at least one set of points for which the projection matrix is sufficiently nonsingular to allow its accurate inversion. Moreover, this redundancy affords considerable opportunities for incorporating explicit noise reduction, if needed, in this zeroth estimate of $\mathbf{u}(\mathbf{x}, t)$.

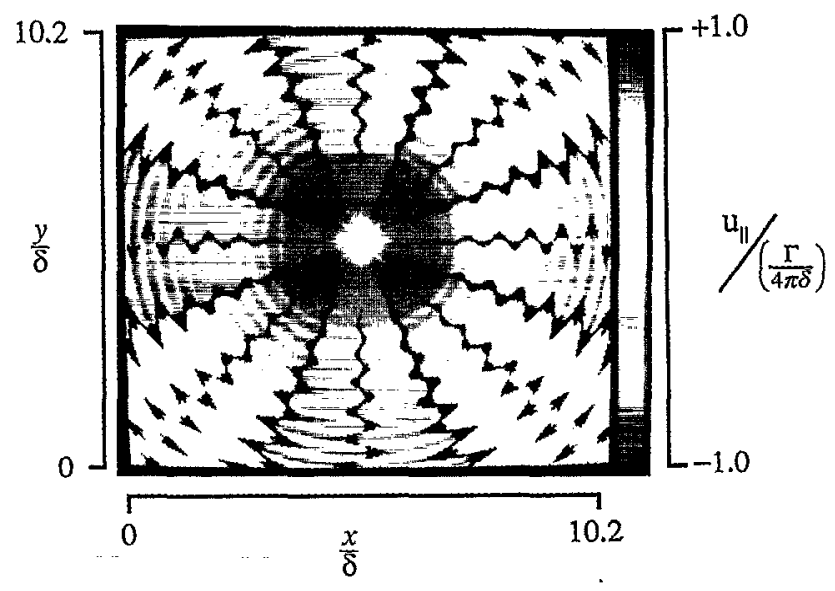

FIG. 3. The velocity component field $u_{\|}(\mathbf{x}, t)$ along the local scalar gradient vector direction $\hat{e}_{\nabla \zeta}(\mathbf{x}, t)$ for Case I in Sec. III $\mathrm{A}$, obtained via $\mathrm{Eq}$ (2).

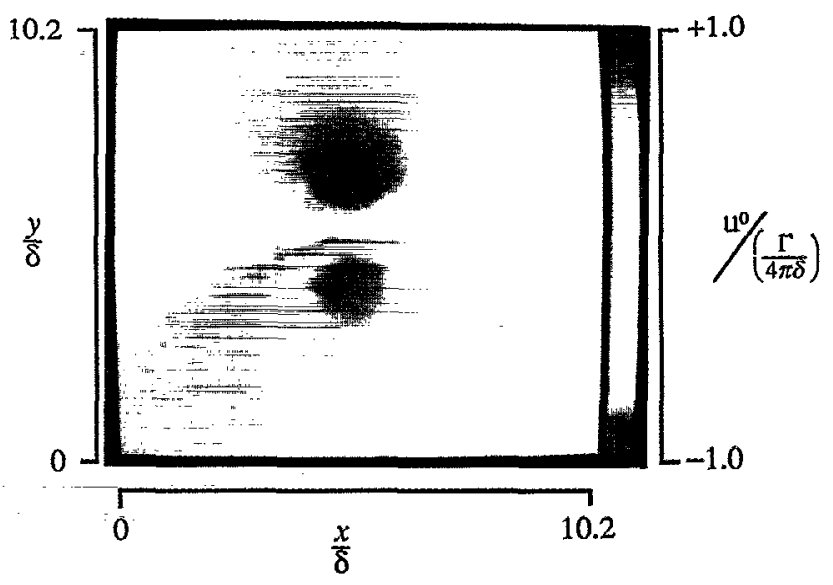

(a)

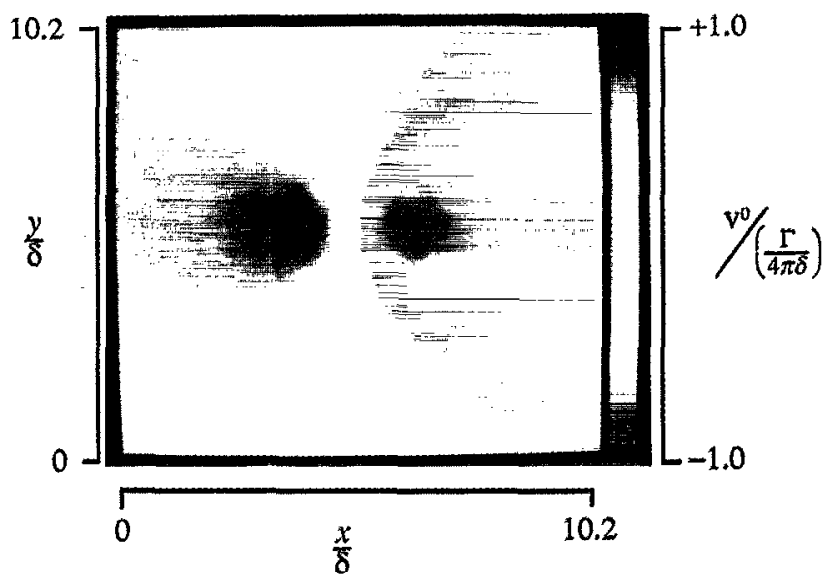

(b)

FIG. 4. The zeroth iteration result $\mathbf{u}^{0}(\mathbf{x}, t)$ for the vector velocity field for Case $I$ in Sec. III A, obtained from the $u_{\|}(x, t)$ field in Fig. 3 via Eq. (6). Compare with the corresponding fourth iteration result $\mathbf{u}^{4}(\mathbf{x}, t)$ in Fig. 5 and the corresponding exact result shown in Fig. 6 . (a) The $x$-component field $u^{0}(\mathrm{x}, t)$. (b) The $y$-component field $v^{0}(\mathrm{x}, t)$. 


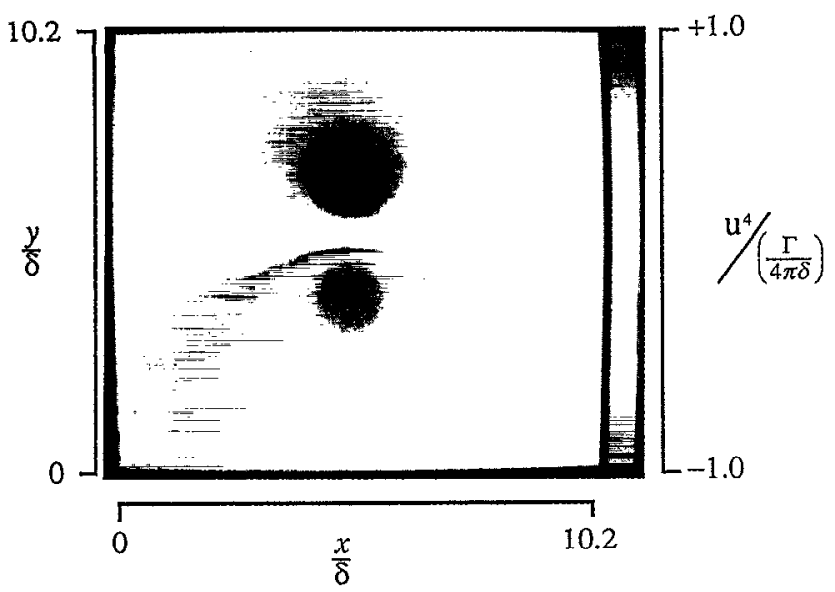

(a)

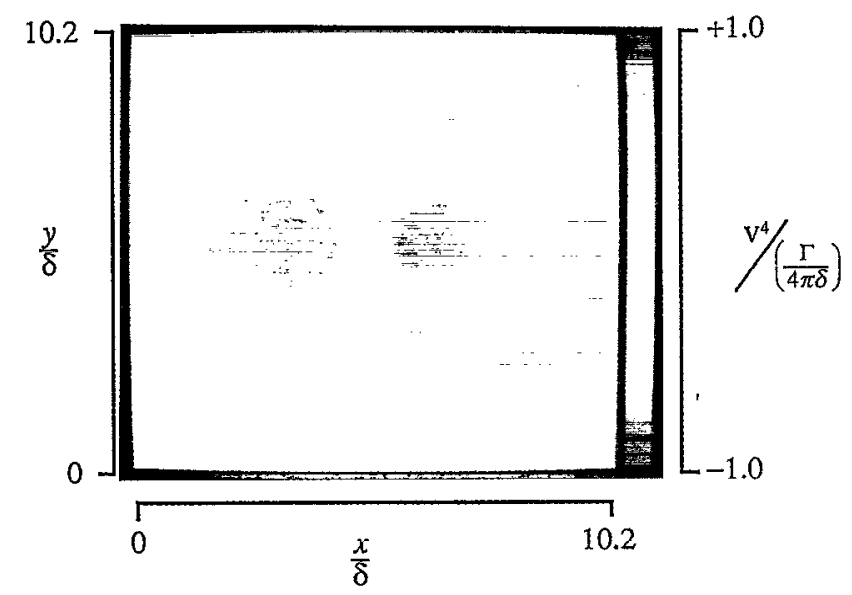

(b)

FIG. 5. The fourth iteration result $\mathbf{u}^{4}(\mathbf{x}, t)$ for the vector velocity field for Case $I$ in Sec. III A, obtained from the zeroth iteration result $\mathbf{u}^{0}(\mathbf{x}, t)$ in Fig. 4 via successive itcrations in Eq. (11). Compare with the zcroth iteration result $\mathbf{u}^{0}(\mathrm{x}, t)$ in Fig. 4 and the corresponding exact result shown in Fig. 6. (a) The $x$-component field $u^{4}(\mathbf{x}, t)$. (b) The $y$-component field $v^{4}(\mathbf{x}, t)$.

Having found the starting values for the velocity vector in regions where the second term in Eq. (4) is negligible, we can then interpolate to obtain a starting solution for $\mathbf{u}(\mathbf{x}, t)$ at each point in the data volume. This starting solution will be denoted by $\mathbf{u}^{0}(\mathbf{x}, t)$. Alternatively, we might obtain the starting solution by simply applying Eq. (6) at every point in each data volume, without attempting to identify those regions where the underlying approximation is most valid. Our results in Sec. III show that, at least for the large Sc considered here, the $\mathbf{u}^{0}(\mathbf{x}, t)$ obtained in this manner are very close to the exact solution.

\section{Successive iterations for $u(x, t)$}

While this starting solution might be adequate for many purposes, it is possible to obtain a more accurate solution for $\mathbf{u}(\mathbf{x}, t)$ by writing Eq. (4) as a sequence of successive approximations, namely

$$
\nabla u_{\|}=\mathbf{u}^{k} \cdot \nabla \hat{e}_{\nabla \zeta}^{T}+\left(\nabla \mathbf{u}^{k-1}\right)^{T} \cdot \hat{e}_{\nabla \zeta}:
$$

Here $\mathbf{u}^{k-1}$ denotes the velocity field obtained from the previous iteration, and $\mathbf{u}^{k}$ the velocity field that results from
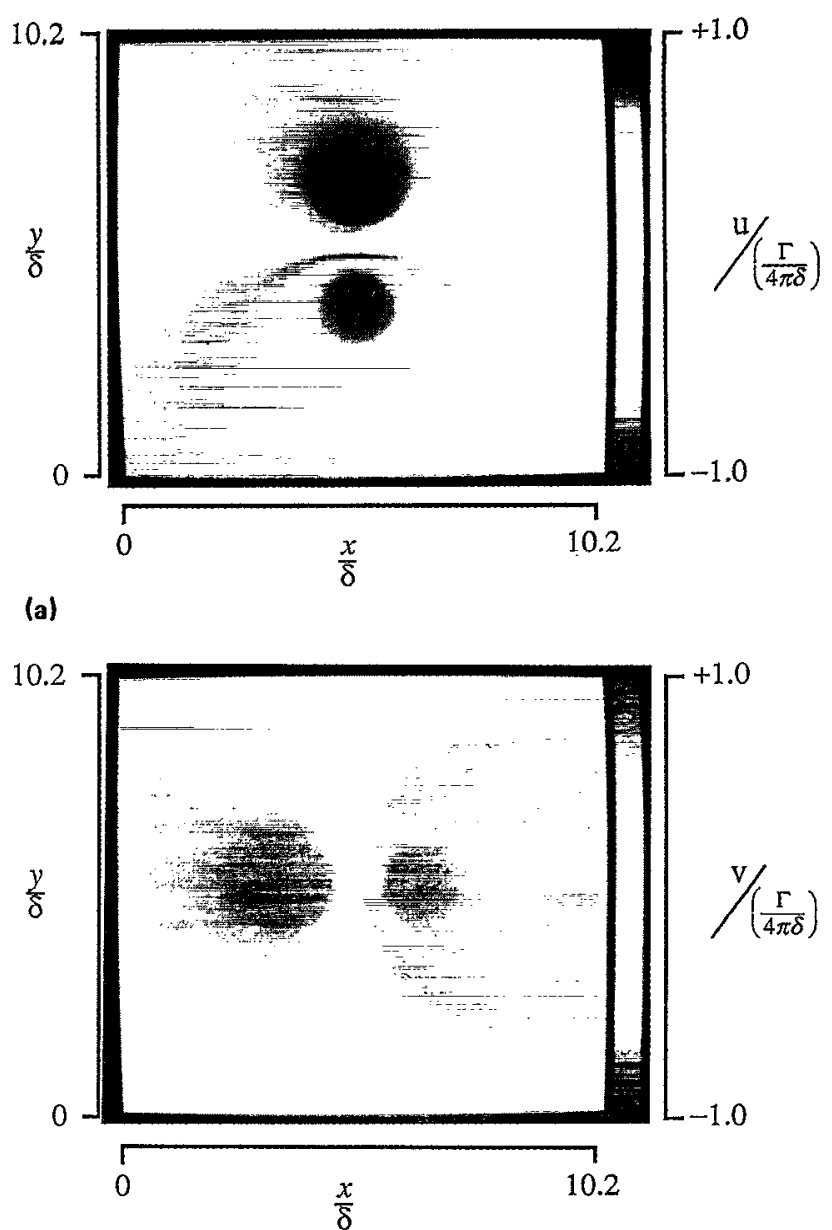

(b)

FIG. 6. The exact analytical result $\mathbf{u}(\mathbf{x}, t)$ for the vector velocity field for Case I in Sec. III A, obtained from Eqs. (9a) and (9b). Compare with the zcroth and fourth itcration results, $\mathbf{u}^{0}(\mathbf{x}, t)$ and $\mathbf{u}^{4}(\mathbf{x}, t)$, in Figs. 4 and 5 , respectively. (a) The $x$-component field $u(x, t)$. (b) The $y$-component field $v(\mathbf{x}, t)$.

using $\mathbf{u}^{k-1}$ to estimate the velocity gradient field $\nabla \mathbf{u}$. We begin the iterative procedure for $k=1$ with the result of the zeroth iteration, $\mathbf{u}^{0}(\mathbf{x}, t)$, from which we find $\nabla \mathbf{u}^{0}(\mathbf{x}, t)$. Equation (7) then involves only the three unknown veloc-

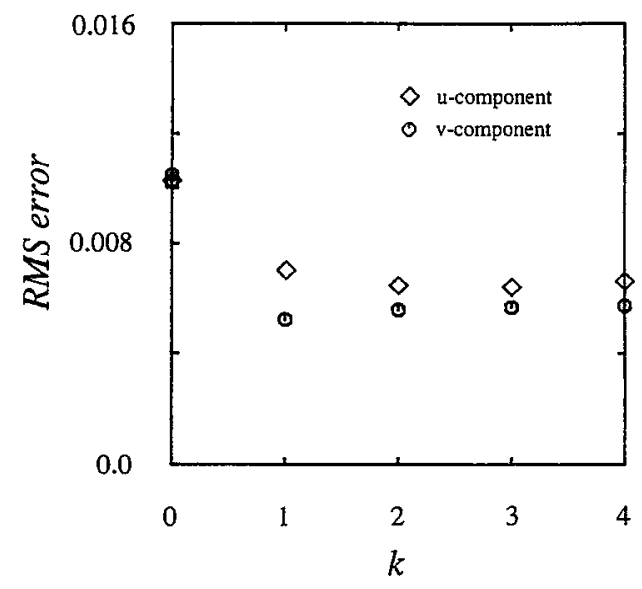

FIG. 7. Reduction in rms velocity errors with increasing iteration number in Eq. (7) for Case I. 

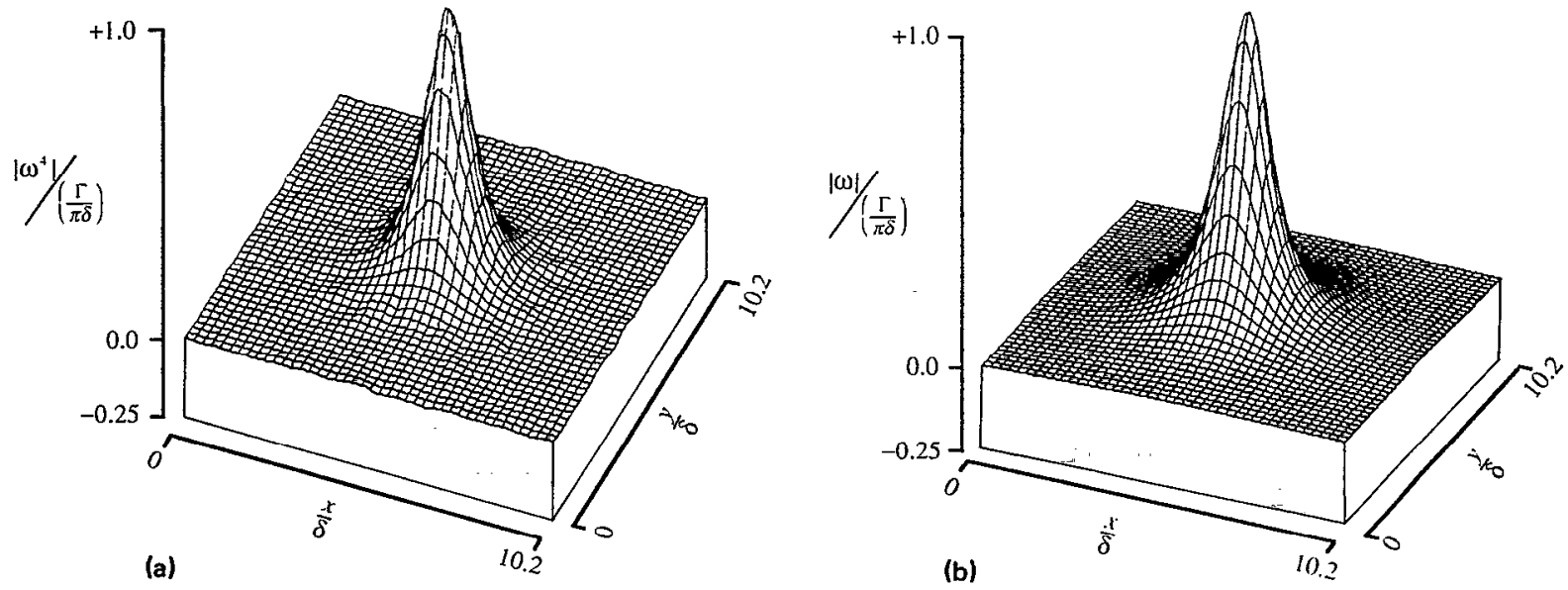

FIG. 8. The vorticity field result $\omega^{4}(\mathbf{x}, t)$ obtained from the fourth iteration result $\mathbf{u}^{4}(\mathbf{x}, t)$ for Case I in Sec. III A, compared with the exact analytical result $\omega(\mathbf{x}, t)$ from Eqs. (9a) and (9b). (a) The resulting $\omega^{4}(\mathbf{x}, t)$ field. (b) The exact $\omega(\mathbf{x}, t)$ field.

ity components $\mathbf{u}^{k}$, which we can obtain from the three components of this equation based on the measured $u_{\|}(\mathbf{x}, t)$ and $\hat{e}_{\nabla \zeta}(\mathbf{x}, t)$ fields. Successive iterations based on Eq. (7) can then be made until the velocity field $\mathbf{u}^{k}$ converges to a self-consistent result. While various constrained iteration schemes that explicitly require $\boldsymbol{\nabla} \cdot \mathbf{u}=0$, or that even enforce the vorticity transport equation explicitly, are possible if needed to accelerate convergence to the velocity field $\mathbf{u}(\mathbf{x}, t)$, our results in the Sec. III show that stable, rapid and accurate convergence is achieved without the need to resort to any such measures.

\section{E. Derivative fields $\epsilon(x, t), \omega(x, t)$, and $\nabla p(x, t)$}

This procedure, in principle, allows the complete space- and time-varying vector velocity field $\mathbf{u}(\mathbf{x}, t)$ to be found throughout the four-dimensional data space. The primary interest is, of course, in its more dynamically insightful derivative fields, namely the three components of the vorticity field $\omega(\mathbf{x}, t) \equiv \nabla \times \mathbf{u}(\mathbf{x}, t)$ and the six components of the strain rate tensor field $\epsilon(\mathbf{x}, t) \equiv \frac{1}{2}\left(\nabla \mathbf{u}+\nabla \mathbf{u}^{T}\right)$. It is also worth noting that the pressure gradient field $\nabla p(\mathbf{x}, t)$ can, in principle, be obtained from $\mathbf{u}(\mathbf{x}, t)$ by inverting the momentum equation as

$$
\nabla p(\mathbf{x}, t)=-\left(\frac{\partial}{\partial t}+\mathbf{u} \cdot \boldsymbol{\nabla}-\frac{1}{\operatorname{Re}} \nabla^{2}\right) \mathbf{u}(\mathbf{x}, t) .
$$

Note that, since the objective velocity field $\mathbf{u}(\mathbf{x}, t)$ is roughly $\mathrm{Sc}^{1 / 2}$ times smoother than the original scalar field measurements, there would appcar to be considerable opportunities for incorporating numerical techniques to insure a twice differentiable result suitable for evaluating $\nabla p(\mathbf{x}, t)$ without compromising the fidelity of the underlying structure of the velocity gradient field $\nabla \mathbf{u}(\mathbf{x}, t)$.

\section{TESTS OF THE SCALAR IMAGING VELOCIMETRY (SIV) TECHNIQUE}

In this section, we apply the scalar imaging velocimetry technique described above to three test cases, with the aim of obtaining a careful validation of the technique and an assessment of its potential accuracy. Case I involves a comparatively simple conserved scalar field $\zeta(\mathrm{x}, t)$ that is defined analytically, and whose time evolution in the presence of a single region of concentrated vorticity is also defined analytically. Since this synthetic conserved scalar field is free of any noise that would be present in an actual measured scalar field, this case provides an assessment of the potential limiting accuracy of the scalar imaging velocimetry method. Moreover, the underlying velocity field $\mathbf{u}(\mathbf{x}, t)$ is known exactly and can be compared with the result obtained from the scalar imaging velocimetry method.

Case II involves an actual measured turbulent flow conserved scalar field $\zeta(\mathbf{x}, t)$, from the fully resolved fourdimensional measurements in Ref. 43 , which is numerically advected in time to give its evolution in the presence of a uniform shear flow. In this case, the scalar field is quite complex and contains a level of noise inherently representative of real turbulent flow measurements. However, the underlying velocity field is still simple and exactly specified, again allowing a precise determination of the accuracy of the technique.

In Case III, the same measured turbulent flow scalar field $\xi(\mathbf{x}, t)$ is advected to give its evolution in the presence of a flow field that is representative of the level of complexity anticipated over the range of inner flow scales spanned by the measurements. The imposed velocity field consists of two vortical structures with circulations of opposite sign and different magnitudes. These three test cases, with the resulting velocity vector fields $\mathbf{u}(\mathbf{x}, t)$, vorticity vector fields $\omega(\mathbf{x}, t)$, and strain rate tensor fields $\epsilon(\mathbf{x}, t)$, are presented in the following sections and compared with the exact values to assess the utility of this scalar imaging velocimetry technique.

\section{A. Case 1}

This case consists of a simple conserved scalar field $\zeta(\mathbf{x}, t)$, shown in Fig. 2, which is numerically evolved un- 


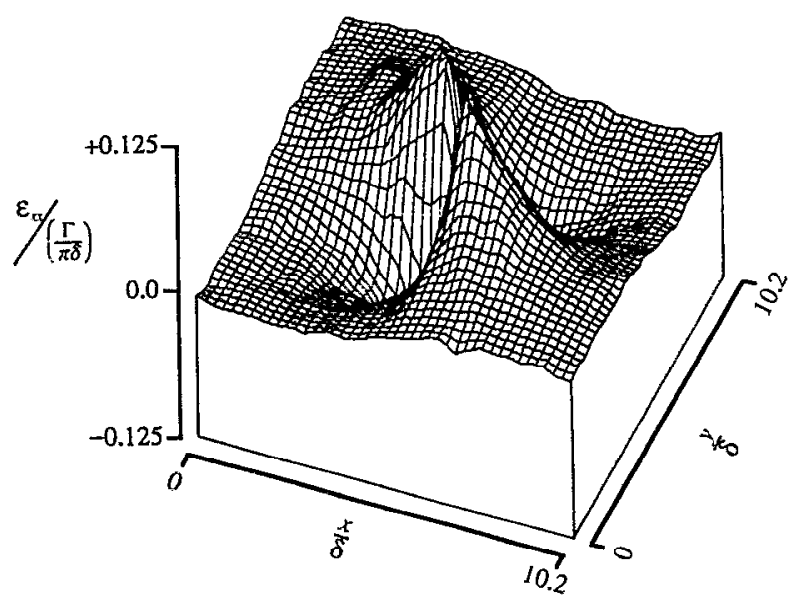

(a)

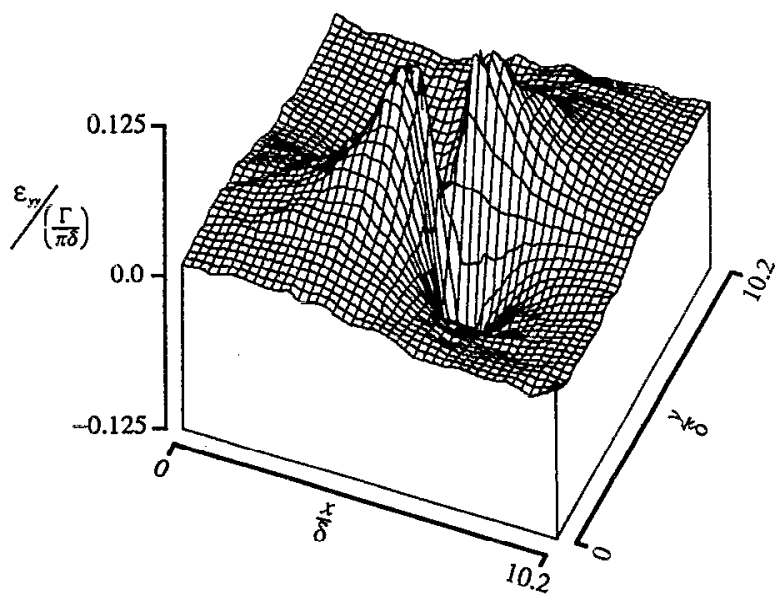

(c)

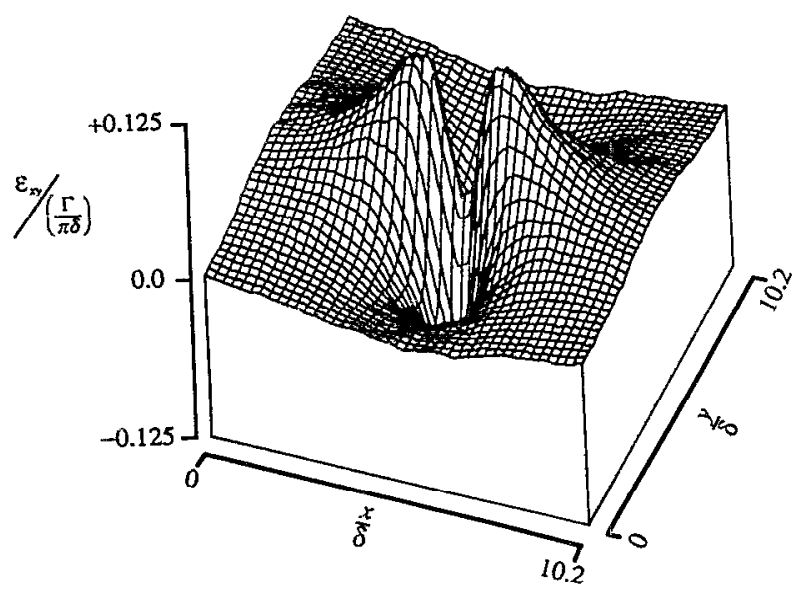

(e)

FIG. 9. The strain rate tensor field result $\epsilon^{4}(\mathbf{x}, t)$ obtained from the fourth iteration result $\mathbf{u}^{4}(\mathbf{x}, t)$ for the vector velocity field for Case I in Sec. III A, compared with the exact analytical result $\epsilon(x, t)$ from Eqs. (9a) and (9b). (a) The resulting $\epsilon_{x x}^{4}(x, t)$ field. (b) The exact $\epsilon_{x x}(x, t)$ fleld. (c) The resulting $\epsilon_{y y}^{4}(\mathbf{x}, t)$ field. (d) The exact $\epsilon_{y y}(\mathbf{x}, t)$ field. (e) The resulting $\epsilon_{x y}^{4}(\mathbf{x}, t)$ field. (f) The exact $\epsilon_{x y}(\mathbf{x}, t)$ field.

der the dynamical influence of a single region of concentrated vorticity. Both the original scalar field and the underlying velocity field are synthetically generated from known analytical functions. As a consequence, there is essentially no noise present in the scalar field from which the velocity field is to be extracted, and thus this case allows an assessment of the limiting accuracy of the method.

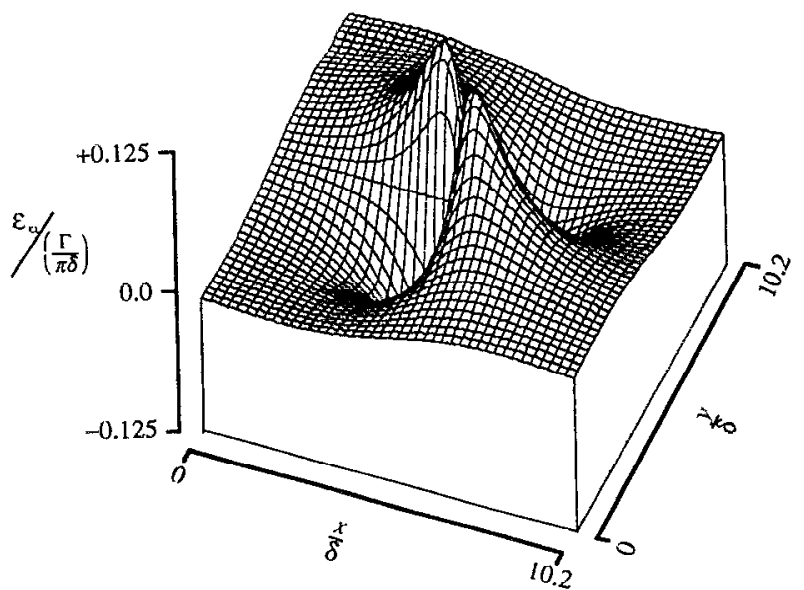

(b)

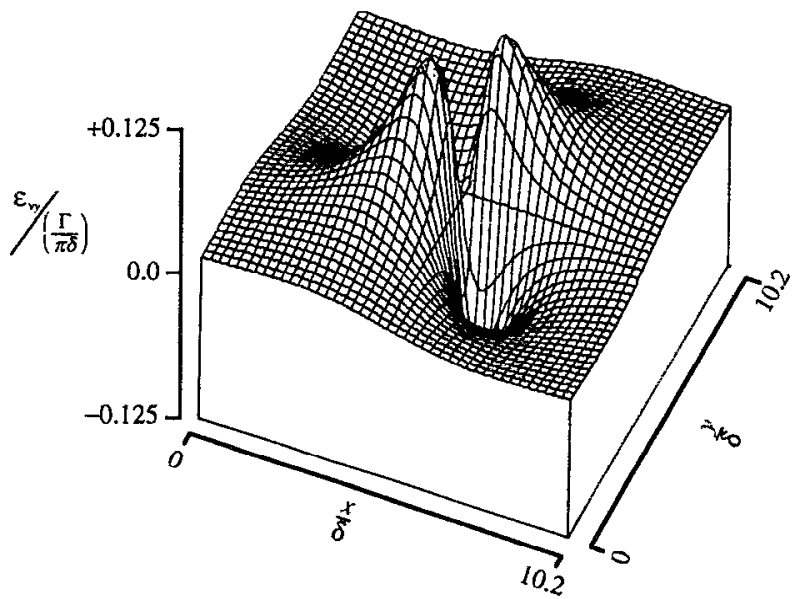

(d)

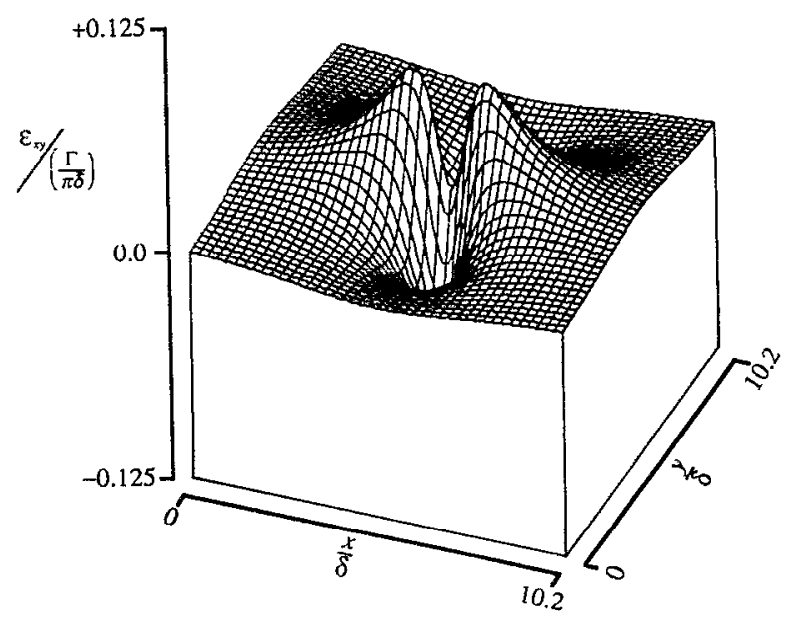

(f)
This scalar field is numerically advanced in time by the velocity field

$$
u(\mathrm{x}, t)=\frac{\Gamma}{2 \pi} \frac{\left(y-y_{0}\right)}{\left(x-x_{0}\right)^{2}+\left(y-y_{0}\right)^{2}+\delta^{2}}
$$

and 


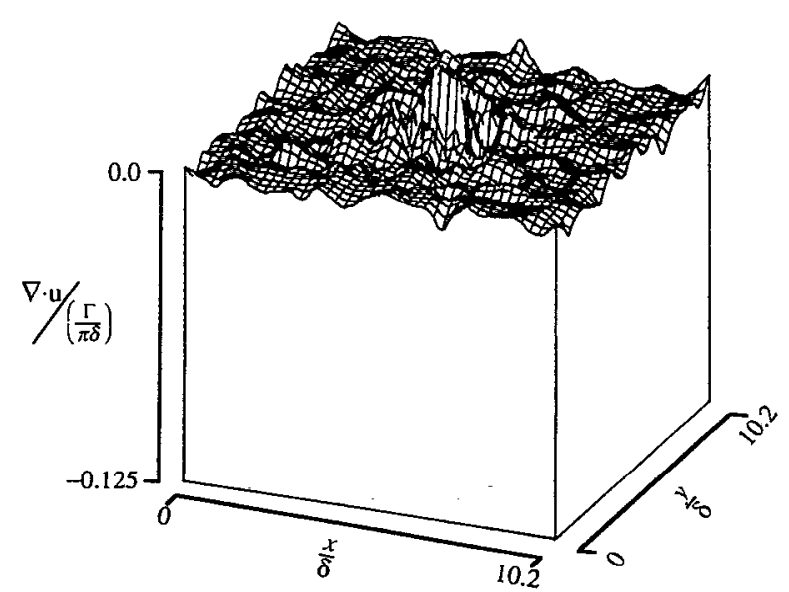

FIG. 10. The trace field resulting from the strain rate tensor field $\epsilon^{4}(\mathbf{x}, t)$ in Fig. 9 for Case I in Sec. III A, shown on the same scale as the strain rate tensor components in Fig. 9 and demonstrating that continuity is essentially satisfied.

$$
v(\mathbf{x}, t)=-\frac{\Gamma}{2 \pi} \frac{\left(x-x_{0}\right)}{\left(x-x_{0}\right)^{2}+\left(y-y_{0}\right)^{2}+\delta^{2}},
$$

which corresponds to a single algebraic vortex blob, located at $\left(x_{0}, y_{0}\right)$ and with circulation $\Gamma$ and core size $\delta$. Normalization of all variables in this case is done with the reference scales $l^{*} \equiv \delta$ and $u^{*} \equiv(\Gamma / 4 \pi \delta)$.

The inversion of the scalar transport equation via $\mathrm{Eq}$. (2) involves the space and time derivatives of the scalar field, which are obtained numerically using central differences from the advected scalar field data (see the Appendix). Equation (2) then produces the $u_{\|}(\mathbf{x}, t)$ field shown in Fig. 3. Note that zero values of $u_{\|}$are indicated by a pure yellow coloring, with colors increasing uniformly to pure red indicating positive values and those increasing to pure blue denoting negative values. Areas in which the scalar gradient vector magnitude lies below a minimum threshold value have been flagged black, since in those

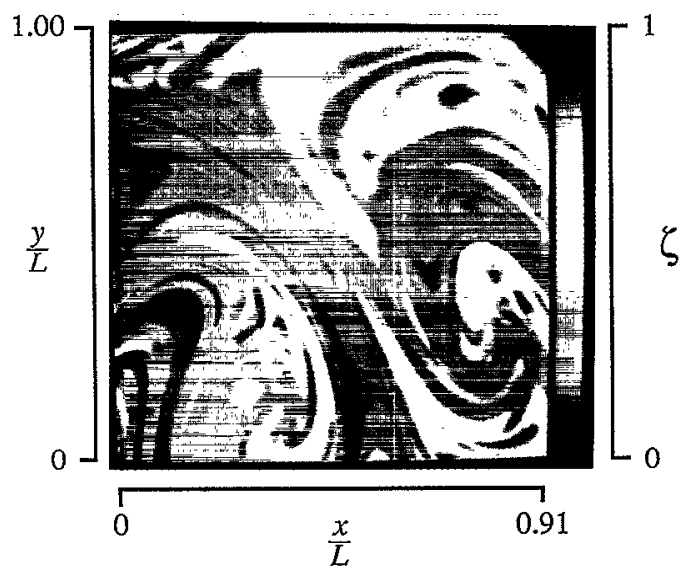

FIG. 11. The conserved scalar field $\zeta(\mathrm{x}, t)$ from the turbulent flow measurements in Ref. 43 used for Case II in Sec. III B.

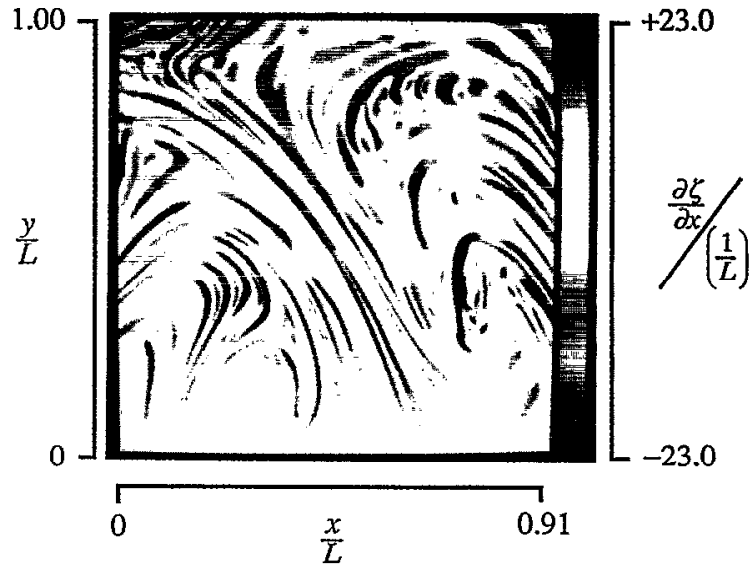

(a)

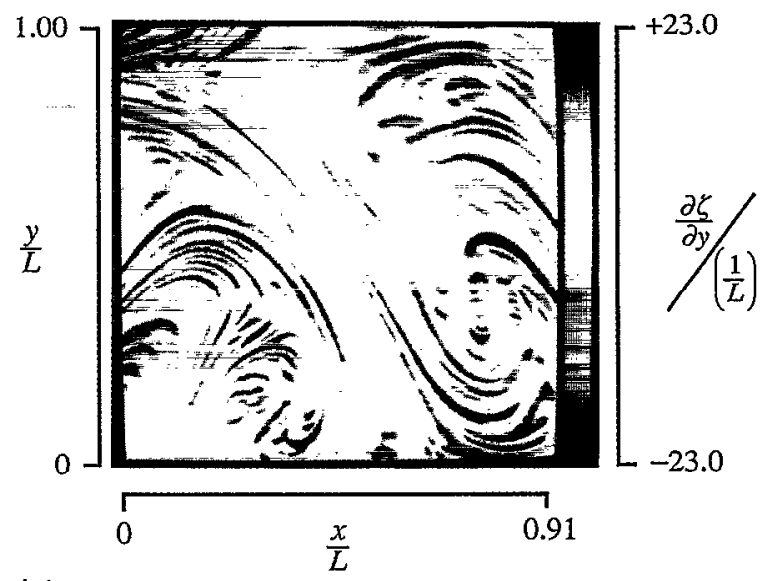

(b)

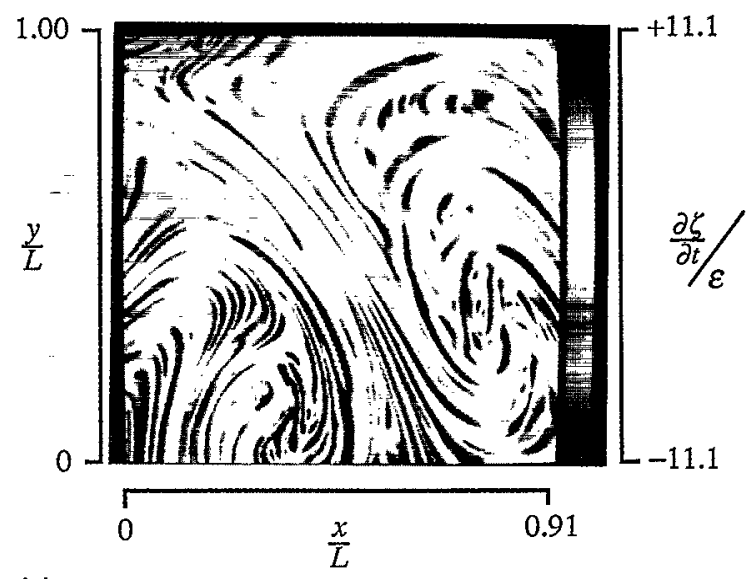

(c)

FIG. 12. The individual derivative fields obtained from the conserved scalar field $\zeta(\mathbf{x}, t)$ in Fig. 11 for Case II in Sec. III B. (a) The $x$-derivative field $(\partial \xi / \partial x)$. (b) The $y$-derivative field $(\partial \zeta / \partial y)$. (c) The $t$-derivative field $(\partial \xi / \partial t)$.

regions no direct inference of $u_{\|}(\mathbf{x}, t)$ from the measured scalar field is possible.

The $u_{\|}(\mathbf{x}, t)$ field in Fig. 3 is then used to extract the zeroth iteration result for the underlying velocity field $\mathbf{u}(\mathbf{x}, t)$ via Eq. (6), which ignores the effect of variations in the true velocity vector $\mathbf{u}$ and approximates variations in $u_{\|}$ as being due solely to variations in $\hat{e}_{\mathbf{v} \zeta}(\mathbf{x}, t)$. The resulting $\mathbf{u}^{0}(\mathbf{x}, t)$ is shown in Fig. 4 , where the same color assign- 


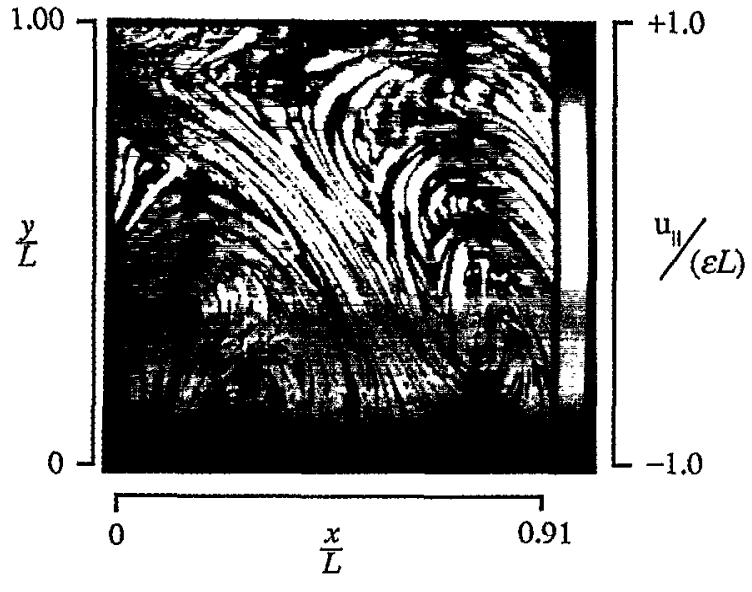

FIG. 13. The velocity component field $u_{\|}(\mathrm{x}, t)$ along the local scalar gradient vector direction for Case II in Sec. III B, obtained from the component fields in Fig. 12 via Eq. (2).

ment scheme as before is used. This initial estimate for the vector velocity field is then used in Eq. (7) to obtain successive improvements in $\mathbf{u}^{k}(\mathbf{x}, t)$. The result obtained after four iterations is shown in Fig. 5, where the improvement in $\mathbf{u}^{4}(\mathbf{x}, t)$ can be seen in comparison with the initial result in Fig. 4. The exact velocity field $\mathbf{u}(\mathbf{x}, t)$ from Eqs. (9a) and $(9 b)$ is shown in Fig. 6, against which the results obtained in Figs. 4 and 5 should be compared. Most of the improvement due to the iterations leading from Figs. 4 to Fig. 5 is actually achieved in the first few iterations. This can be seen in Fig. 7, which shows the reduction of the rms error in each velocity component, normalized by the peak value of that component, with increasing iteration number $k$.

The vorticity field $\omega(\mathbf{x}, t)$ obtained from the fourth iteration result $\mathbf{u}^{4}(\mathbf{x}, t)$ in Fig. 5 is shown in Fig. 8(a), with the corresponding exact result from Eqs. (9a) and (9b)

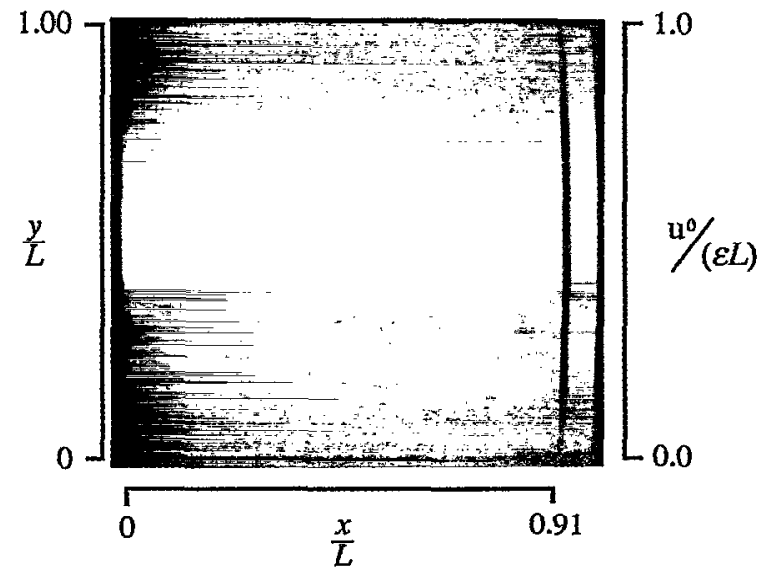

FIG. 14. The zeroth iteration result $\mathbf{u}^{0}(\mathbf{x}, t)$ for the vector velocity field for Case II in Sec. III B, obtained from the $u_{\|}(\mathrm{x}, t)$ field in Fig. 13 via Eq. (6). Compare with the corresponding fourth itcration result $\mathbf{u}^{4}(\mathbf{x}, t)$ in Fig. 15 and the corresponding exact result shown in Fig. 16. (a) The $x$-component field $u^{0}(\mathbf{x}, t)$. (b) The $y$-component field $v^{0}(\mathbf{x}, t)$.

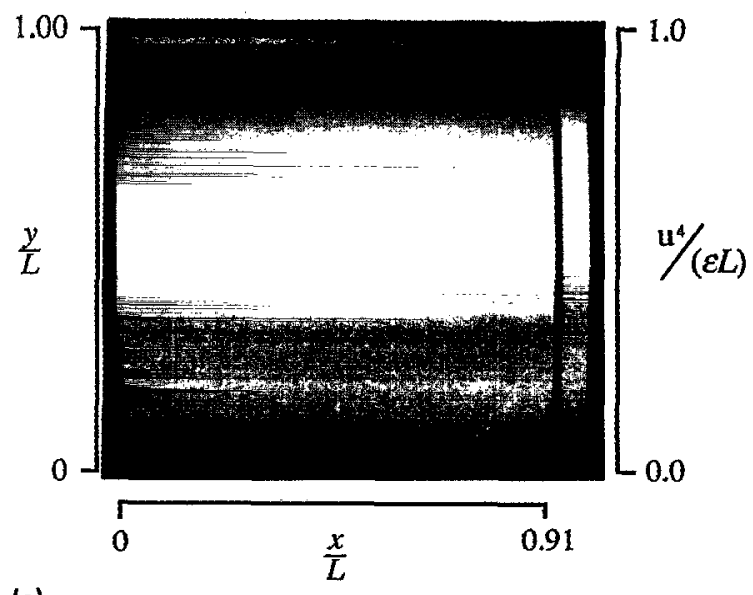

(a)

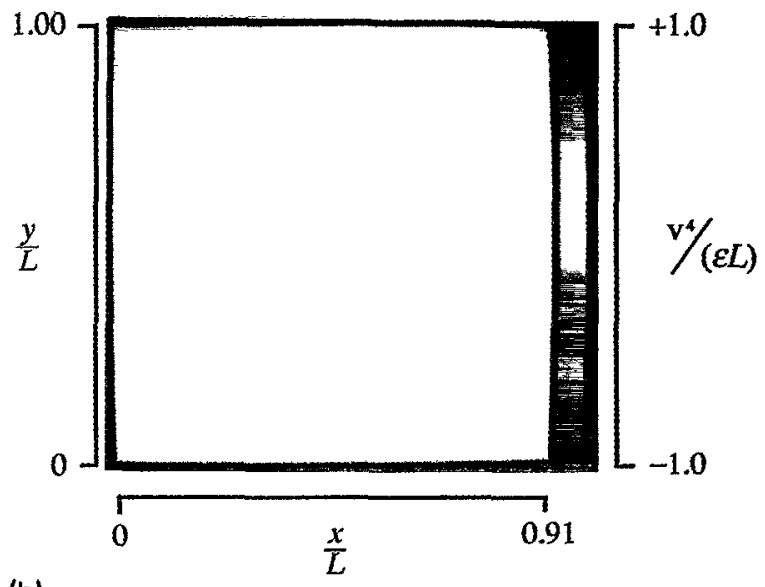

(b)

FIG. 15. The fourth iteration result $\mathbf{u}^{4}(\mathbf{x}, t)$ for the vector velocity field for Case II in Sec. III B, obtained from the zeroth iteration result $\mathbf{u}^{0}(\mathrm{x}, t)$ in Fig. 14 via successive iterations in Eq. (11). Compare with the zeroth iteration result $u^{0}(\mathbf{x}, t)$ in Fig. 14 and the corresponding exact result shown in Fig. 16. (a) The $x$-component field $u^{4}(\mathrm{x}, t)$. (b) The $y$ component field $v^{4}(\mathbf{x}, t)$.

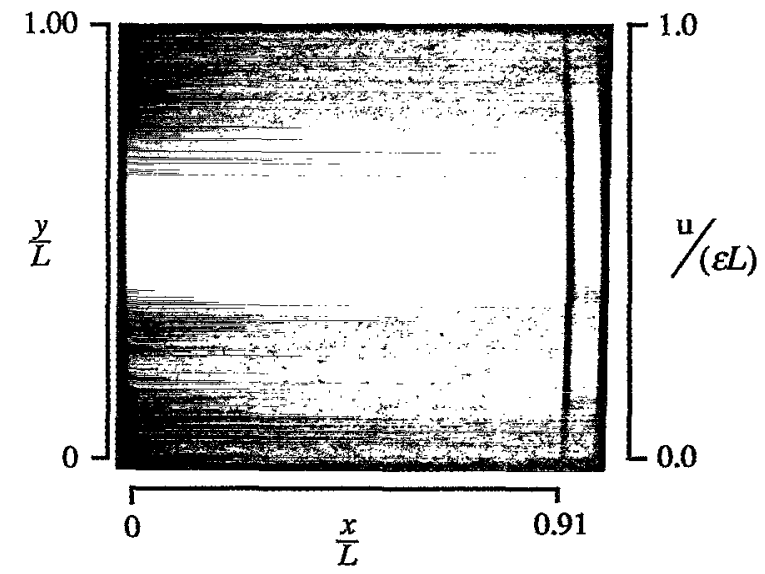

FIG. 16. The exact analytical result $u(\mathbf{x}, t)$ for the velocity field in Case II of Sec. III B. Note that the exact result for $v(\mathbf{x}, t)$ is identically zero. Compare with the zeroth and fourth iteration results, $\mathbf{u}^{0}(\mathbf{x}, t)$ and $\mathbf{u}^{4}(\mathbf{x}, t)$, in Figs. 14 and 15, respectively. 


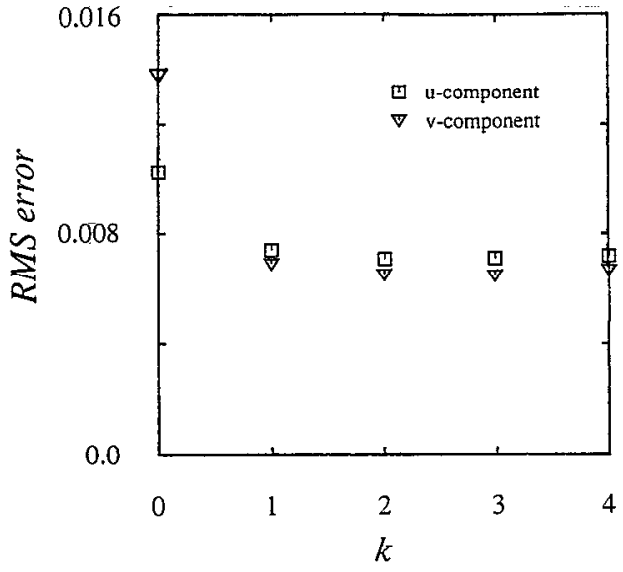

FIG. 17. Reduction in rms velocity errors with increasing iteration number in Eq. (7) for Case II.

shown for comparison in Fig. 8(b). Similarly, the three independent components of the strain rate tensor field $\boldsymbol{\epsilon}(\mathbf{x}, t)$ obtained from the fourth iteration result $\mathbf{u}^{4}(\mathbf{x}, t)$ are compared with the exact results in Fig. 9. Note that both the vorticity and strain rate fields are very accurately extracted by the technique. As a final and rather stringent test of the accuracy of these results, Fig. 10 shows the trace field resulting from the strain rate tensor field $\epsilon(x, t)$ obtained from $\mathbf{u}^{4}(\mathbf{x}, t)$; the scale is the same as those in Fig. 9. The result in Fig. 11 verifies that continuity is well satisfied by the vector velocity field obtained from this technique.

\section{B. Case II}

This case addresses the use of measured conserved scalar field data $\zeta(\mathbf{x}, t)$ from an actual turbulent flow in this scalar imaging velocimetry technique. The scalar field used is from the fully resolved four-dimensional turbulent flow measurements in Ref. 43, and is shown in Fig. 11. This scalar field is advected in time using a second-order Runge-Kutta scheme to give its evolution in the presence

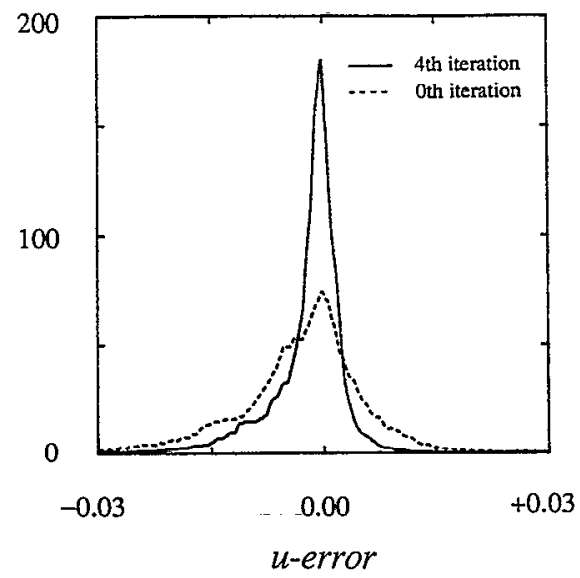

FIG. 18. Distributions of $u(x, t)$ velocity component errors with increasing iteration number $k$ for Case II.

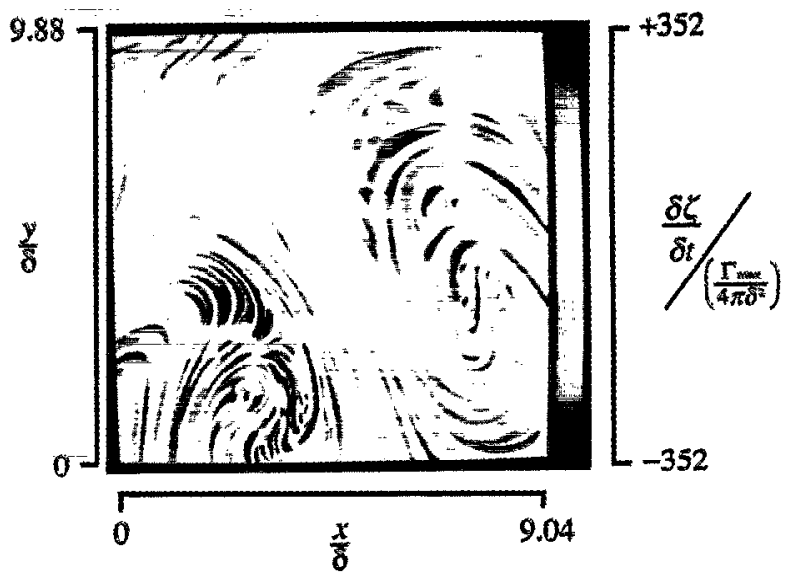

FIG. 19. The time derivative field $(\partial \xi / \partial t)$ obtained from the measured turbulent flow conserved scalar field $\xi(\mathbf{x}, t)$ in Fig. 11 for Case III in Sec. III $\mathrm{C}$. The $x$-derivative and $y$-derivative fields for this case are essentially identical to those shown in Figs. 12(a) and 12(b).

of a uniform shear flow with $\nu \equiv 0$ and with velocity gradient $(\partial u / \partial y) \equiv \epsilon$. In this case, normalization of all variables is done with the reference scales $l * \equiv L$ and $u^{*} \equiv(\epsilon L)$. While the velocity field in this case is still relatively simple, the scalar field is inherently representative of real turbulent flow scalar fields, and, moreover, contains a level of noise that also is inherently representative of real scalar imaging measurements in turbulent flows. Again, the fact that the velocity field is known analytically allows precise assessments of the accuracy of the method for this considerably more complex case.

We begin by examining the integrity of the derivative fields involved in Eq. (2), which are shown in Fig. 12. The same color assignment scheme as before is used to denote the quantitative derivative values. Despite the noise inherent in the measured turbulent flow scalar field data, the derivative fields obtained are relatively smooth. From these, the inversion in Eq. (2) produces the $u_{\|}(\mathrm{x}, t)$ field

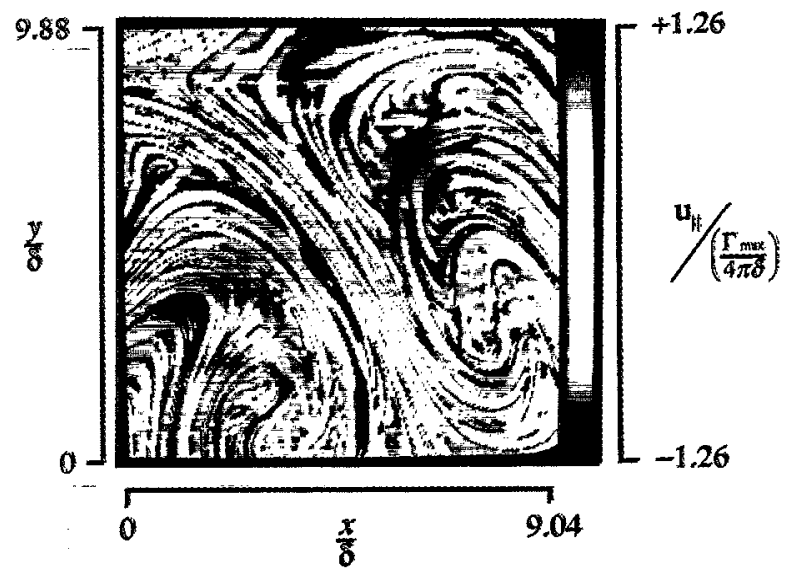

FIG. 20. The velocity component field $u_{\|}(\mathrm{x}, t)$ along the local scalar gradient vector direction $\hat{e}_{\nabla 5}(x, t)$ for Case III in Sec. III C, obtained via Eq. (2). 


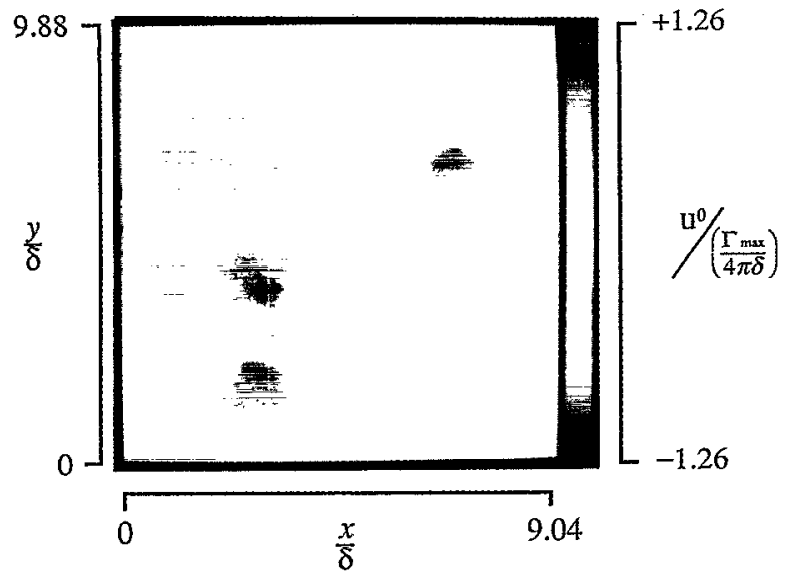

(a)

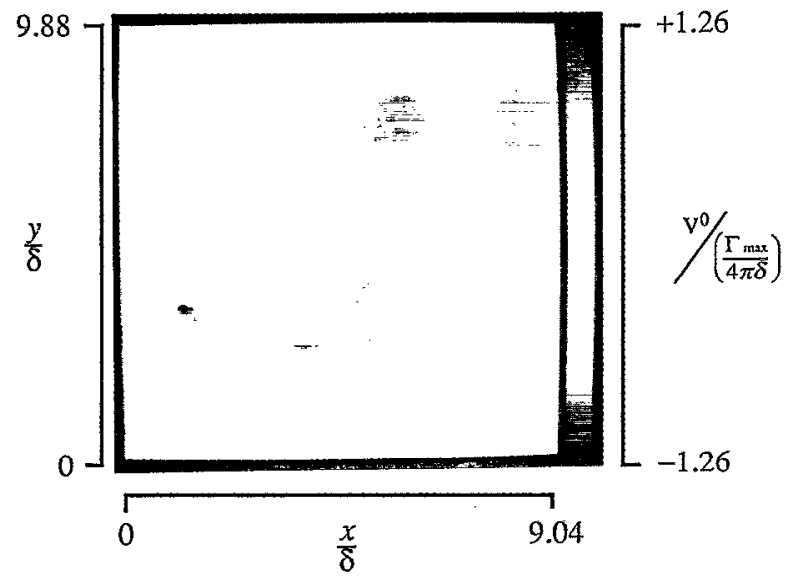

(b)

FIG. 21. The zeroth iteration result $\mathbf{u}^{0}(\mathbf{x}, t)$ for the vector velocity field for Case III in Sec. III C, obtained from the $u_{\mathrm{H}}(\mathrm{x}, t)$ field in Fig. 20 via Eq. (6). Compare with the corresponding fourth iteration result $\mathbf{u}^{4}(\mathbf{x}, t)$ in Fig. 22 and the corresponding exact result shown in Fig. 23. (a) The $x$-component field $u^{0}(\mathrm{x}, t)$. (b) The $y$-component field $v^{0}(\mathbf{x}, t)$.

shown in Fig. 13. Note that the layerlike structure inherent in the scalar gradient vector field $\nabla \xi(x, t)$ leads to both the structure and the color symmetry seen in $u_{\|}(\mathbf{x}, t)$ in Fig. 13.

The zeroth iteration result $\mathbf{u}^{0}(\mathbf{x}, t)$ obtained via Eq. (6) is shown in Fig. 14. The result obtained after four iterations of Eq. (7) based on this initial result is shown in Fig. 15. The improvement in $\mathbf{u}^{4}(\mathbf{x}, t)$ can be seen by comparing with the initial result in Fig. 14 and with the exact velocity field $\mathbf{u}(\mathbf{x}, t)$ shown in Fig. 16. For this uniform shear case, the vorticity field $\omega(\mathbf{x}, t)$ and strain rate tensor field $\epsilon(\mathbf{x}, t)$ are uniform, and thus, for brevity, will not presented here. As in the previous case, Fig. 17 shows that most of the improvement between $\mathbf{u}^{0}(\mathbf{x}, t)$ and $\mathbf{u}^{4}(\mathbf{x}, t)$ is obtained in the first few iterations. Figure 18 shows this convergence in terms of the entire distribution of $u$-component errors, where again the error is normalized by the peak value of the true velocity component.

\section{Case III}

We now consider the same measured turbulent flow scalar field shown in Fig. 11, but impose a somewhat more

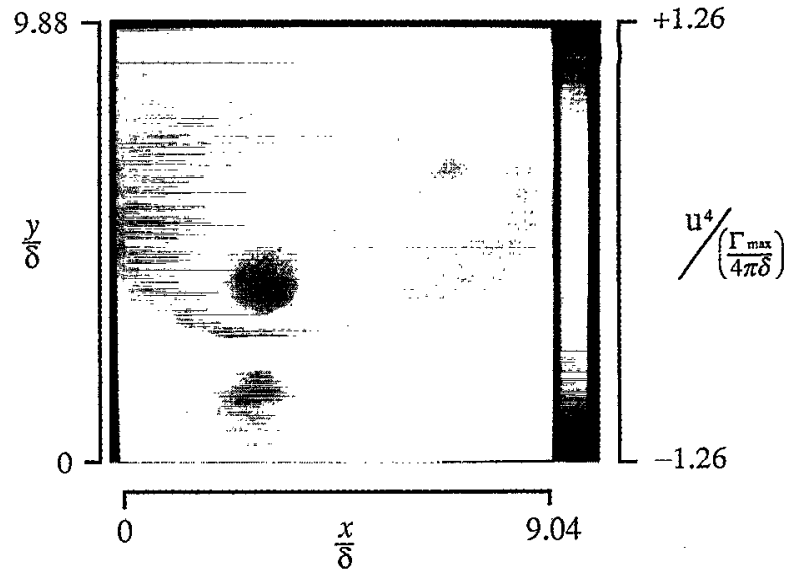

(a)

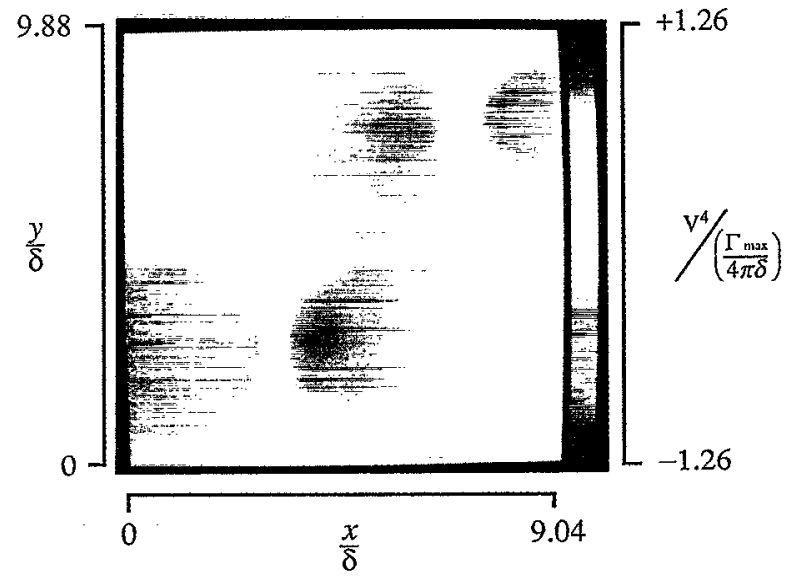

(b)

FIG. 22. The fourth iteration result $\mathbf{u}^{4}(\mathbf{x}, t)$ for the vector velocity field for Case III in Sec. III C, obtained from the zeroth iteration result $\mathbf{u}^{0}(\mathbf{x}, t)$ in Fig. 21 via successive iterations in Eq. (11). Compare with the zeroth iteration result $\mathbf{u}^{\mathrm{U}}(\mathbf{x}, t)$ in Fig. 21 and the corresponding exact result shown in Fig. 23. (a) The $x$-component field $u^{4}(\mathrm{x}, t)$. (b) The $y$ component field $v^{4}(\mathbf{x}, t)$.

complex velocity field. The velocity gradients are now considerably higher and are no longer spatially uniform, with the velocity field being representative of the level of complexity anticipated over the range of inner flow scales spanned by the measured turbulent flow scalar field data. In particular, we use

$$
u(\mathbf{x}, t)=\sum_{i=1}^{2} \frac{\Gamma_{i}}{2 \pi} \frac{\left(y-y_{i}\right)}{\left(x-x_{i}\right)^{2}+\left(y-y_{i}\right)^{2}+\delta_{i}^{2}}
$$

and

$$
v(\mathbf{x}, t)=-\sum_{i=1}^{2} \frac{\Gamma_{i}}{2 \pi} \frac{\left(x-x_{i}\right)}{\left(x-x_{i}\right)^{2}+\left(y-y_{i}\right)^{2}+\delta_{i}^{2}},
$$

which corresponds to two vortex blobs of the type in Case I. Here, we take the circulation ratio $\Gamma_{1} / \Gamma_{2}=-3 / 2$ and $\delta_{2} / \delta_{1}=1$. Normalization in this case is done with the reference scales $l^{*} \equiv \delta$ and $u^{*} \equiv\left(\Gamma_{1} / 4 \pi \delta\right)$. The dimensionless data plane size was 10 , and the vortex locations were chosen as $\left(x_{1}, y_{1}\right)=(3,3)$ and $\left(x_{2}, y_{2}\right)=(7,8)$. The same measured turbulent flow scalar field as in Case II was advected 


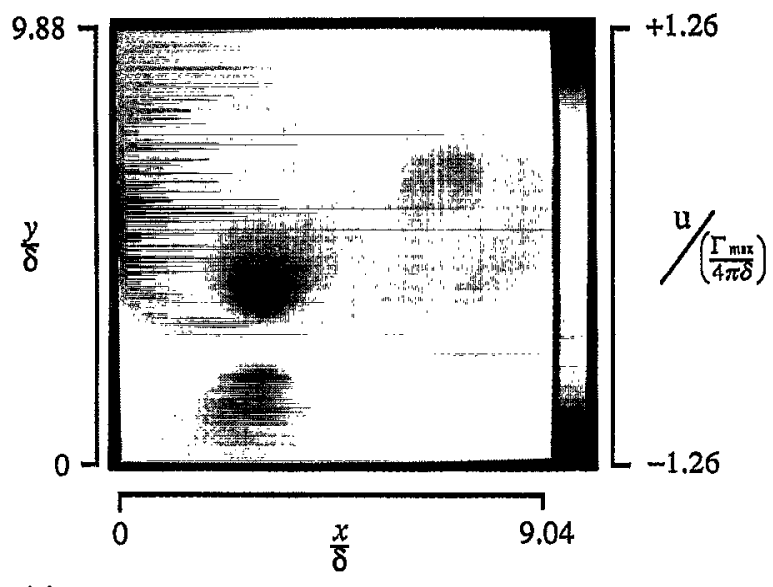

(a)

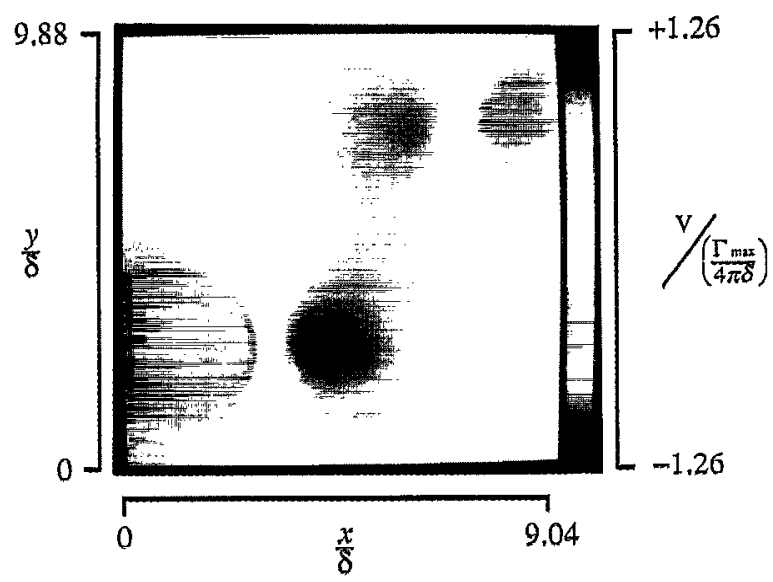

(b)

FIG. 23. The exact analytical result $\mathbf{u}(\mathbf{x}, t)$ for the vector velocity field for Case III in Sec. III C, obtained from Eqs. (10a) and (10b). Compare with the zeroth and fourth iteration results, $\mathbf{u}^{0}(\mathbf{x}, t)$ and $\mathbf{u}^{4}(\mathbf{x}, t)$, in Figs. 21 and 22 , respectively. (a) The $x$-component field $u(x, t)$. (b) The $y$ component field $v(\mathrm{x}, t)$.

in time with a second-order Runge-Kutta scheme to produce the corresponding scalar fields at different times.

The space and time derivatives of the scalar field involved in Eq. (2) were again obtained from the advected

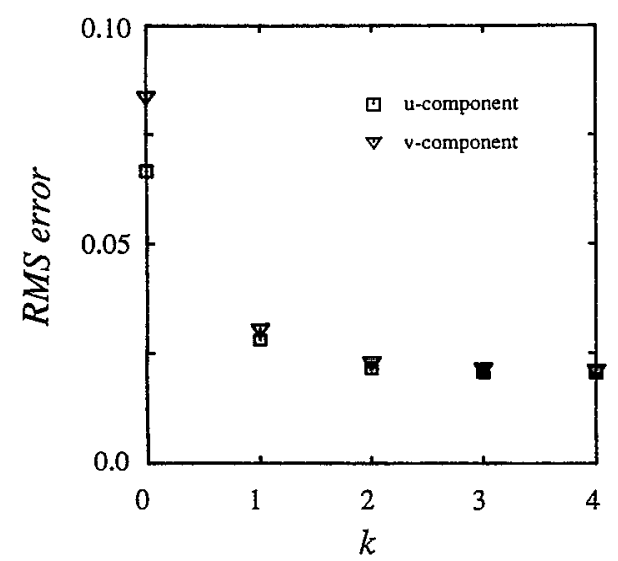

FIG. 24. Reduction in rms velocity errors with increasing iteration number in Eq. (7) for Case III.
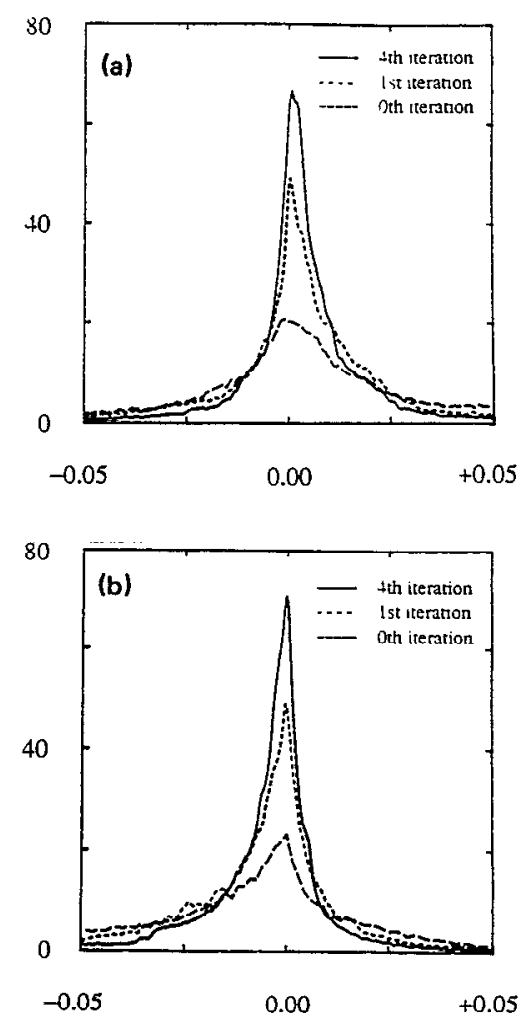

FIG. 25. Distributions of the velocity component errors with increasing iteration number for Case III. (a) $u$-component errors. (b) $v$-component errors.

scalar field data. The spatial derivatives are essentially identical to those in Figs. 12(a) and 12(b) but the time derivative is now different owing to the different flow field and is shown in Fig. 19. From these derivative fields, the inversion in Eq. (2) produces the $u_{\|}(\mathbf{x}, t)$ field shown in Fig. 20.

The zeroth iteration result $\mathbf{u}^{0}(\mathbf{x}, t)$ from $\mathrm{Eq} .(6)$ is shown in Fig. 21. The corresponding result for $\mathbf{u}^{4}(\mathbf{x}, t)$ after four iterations of Eq. (7) is shown in Fig. 22. The exact velocity field $\mathbf{u}(\mathbf{x}, t)$ from Eqs. (10a) and (10b) is shown in Fig. 23, against which the results obtained in Figs. 21 and 22 should be compared. The $\mathbf{u}^{4}(\mathbf{x}, t)$ field can be clearly seen to be an improvement over the initial field $\mathbf{u}^{0}(\mathbf{x}, t)$, and moreover can be seen to compare well with the exact $\mathbf{u}(\mathbf{x}, t)$. The convergence is summarized in Figs. 24 and 25 , which show the reduction in rms velocity component errors as well as the entire distribution of velocity errors.

The vorticity field $\omega(\mathbf{x}, t)$ obtained from the $\mathbf{u}^{4}(\mathbf{x}, t)$ field in Fig. 22 is shown in Fig. 26(a), and should be compared with the corresponding exact result from Eqs. (10a) and (10b) shown in Fig. 26(b). Similarly, the three independent components of the strain rate tensor field $\epsilon(\mathbf{x}, t)$ obtained from the $\mathbf{u}^{4}(\mathbf{x}, t)$ field are compared with the corresponding exact results in Fig. 27. Note that all the major structural features of the vorticity and strain rate fields are accurately reproduced even in this more complex case. As a final check on the accuracy of the resulting 


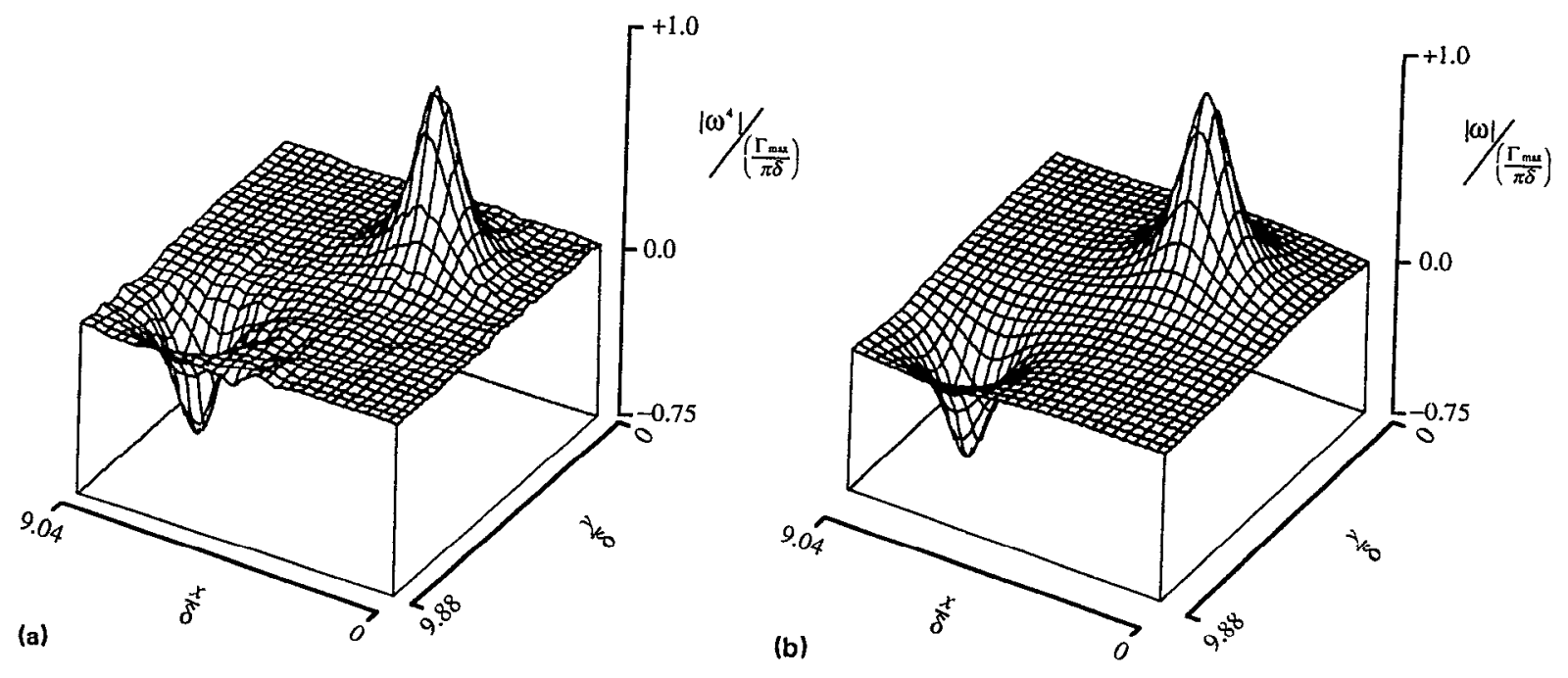

FIG. 26. The vorticity field result $\omega^{4}(\mathbf{x}, t)$ obtained from the fourth iteration result $\mathbf{u}^{4}(\mathbf{x}, t)$ for the vector velocity field for Case III in Sec. III C, compared with the exact analytical result $\omega(\mathbf{x}, t)$ from Eqs. (10a) and (10b). (a) The resulting $\omega^{4}(\mathbf{x}, t)$ field. (b) The exact $\omega(\mathbf{x}, t)$ field.

$\mathbf{u}^{4}(\mathbf{x}, t)$ field, Fig. 28 shows the trace field obtained from the strain rate tensor field $\epsilon(x, t)$ in Figs. 27(a) and 27(c). The scale is the same as those in Fig. 27 and verifies that continuity is again essentially satisfied by the resulting vector velocity field obtained from this scalar imaging velocimetry technique.

\section{CONCLUSIONS}

We have presented an experimental technique for extracting the underlying vector velocity field throughout a four-dimensional spatiotemporal region from fully resolved measurements of a conserved scalar field. The technique has been applied to a series of test cases specifically selected to assess the validity and potential accuracy of the technique through detailed comparisons against known velocity fields. In particular, the results from Case I, which involves essentially no noise in the original scalar field data, suggest that highly accurate extraction of the underlying vorticity and strain rate fields is possible with this approach. Moreover, since Cases II and III involve actual measured turbulent flow scalar fields, and since the velocity gradient length scale $\lambda_{v}$ in Case III reflects a level of complexity in the velocity field relative to the scalar gradient length scale $\lambda_{D}$ that is representative of actual turbulent flows, results from these tests should be indicative of the applicability of the technique to the study of turbulent flows. The spatial and temporal resolution, as well as the noise level, of the original scalar field measurements appear to be the major practical issues in applying the method. In this context, we note that the measurement of sufficiently well-resolved scalar fields has been reported in Ref. 43, and, indeed, these measurements have been used for two of the present test cases. Based on these test cases, it appears that current imaging technology allows the requisite scalar field to be measured with sufficiently high integrity to permit the inversion described here. It is important to note in this regard that, for a given signal-to-noise level, measure- ment of the scalar field in all three spatial dimensions offers considerably greater noise reduction advantages in computing derivatives than experience in more traditional onedimensional measurements might suggest. For example, a properly defined central difference operator on a full $3^{3}$ local spatial template produces a gradient field with far less noise and with the same spatial resolution than a naive template involving just six points would for the same noise level in the original scalar field. The time derivative, however, offers no such noise reduction possibilities. Accordingly, our experience to date has shown that noise in this term is functionally the limiting quantity in performing the inversion. The results from Cases II and III nevertheless suggest that measurements such as these are capable of producing sufficiently low noise data to allow the inversion to determine the underlying velocity field. Collectively, the results obtained suggest that this scalar imaging velocimetry technique is capable of yielding fully resolved experimental data for the space- and time-varying vector velocity field, and the associated structure and dynamics of its derivative fields, on the inner scales of turbulent flows.

It is striking that the initial estimates $\mathbf{u}^{0}(\mathbf{x}, t)$ are as good as they are, and that most of the successive improvement from Eq. (7) occurs in the first iterative step. The former appears to be due to the fact that the initial neglect of $\boldsymbol{\nabla u} \cdot \hat{e}_{\nabla \zeta}$ in favor of $\mathbf{u} \cdot \nabla \hat{e}_{\nabla \zeta}$ in Eq. (4) is indeed a good approximation at large Schmidt numbers. The latter may then result from the fact that, given this good initial estimate, the iteration in Eq. (7) is able to essentially correct for the errors introduced by this approximation on the first step. The ultimate level of improvement attainable through further iterations is, of course, limited by numerical processing errors introduced by the discrete implementation of Eq. (7).

While the technique appears to work well for $\mathrm{Sc} \gg 1$ scalar fields, it is interesting to consider whether the same can be expected for $S c \approx 1$ scalar fields. In the latter case, 

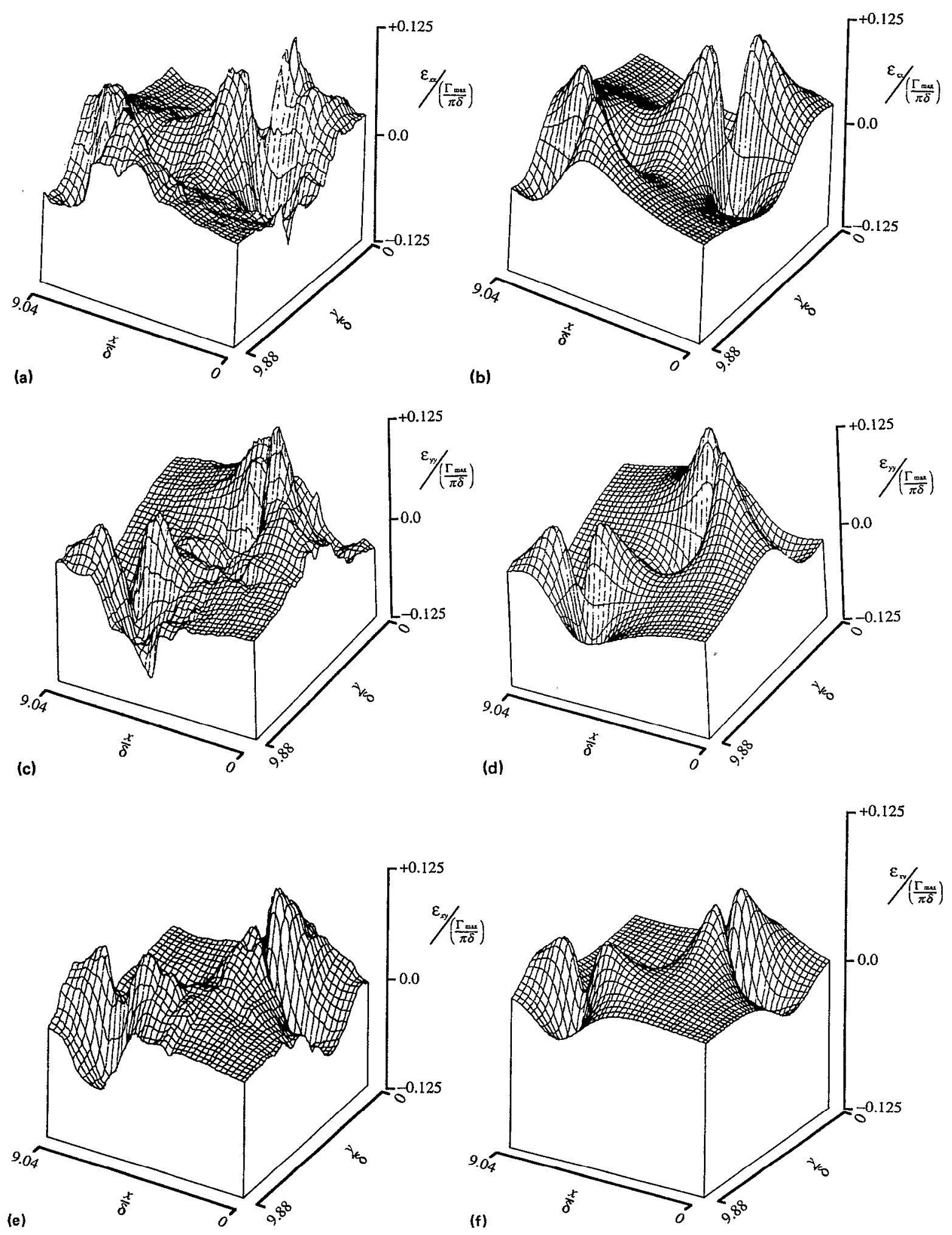

FIG. 27. The strain rate tensor field result $\epsilon^{4}(\mathbf{x}, t)$ obtained from the fourth iteration result $\mathbf{u}^{4}(\mathbf{x}, t)$ for the vector velocity field for Case III in Sec. III C, compared with the exact analytical result $\epsilon(\mathbf{x}, t)$ from Eqs. (10a) and (10b). (a) The resulting $\epsilon_{x x}^{4}(\mathbf{x}, t)$ field. (b) The exact $\epsilon_{x x}(\mathbf{x}, t)$ field. (c) The resulting $\epsilon_{y y}^{4}(\mathbf{x}, t)$ field. (d) The exact $\epsilon_{y y}(\mathbf{x}, t)$ field. (e) The resulting $\epsilon_{x y}^{4}(\mathbf{x}, t)$ field. (f) The exact $\epsilon_{x y}(\mathbf{x}, t)$ field.

the assumption that the first term on the right in Eq. (4) will be small in comparison with the second will be poorer. However, that may only lead to a poorer initial estimate $\mathbf{u}^{0}(\mathbf{x}, t)$. The properties of the iteration in Eq. (7) suggest that $\mathbf{u}^{k}(\mathbf{x}, t)$ should still converge to the correct $\mathbf{u}(\mathbf{x}, t)$, though this will likely require more iterations. Note that measurement techniques for $\mathrm{Sc} \approx 1$ scalar fields comparable to those in Refs. 43 and 44 for $S c \gg 1$ scalars are not yet 


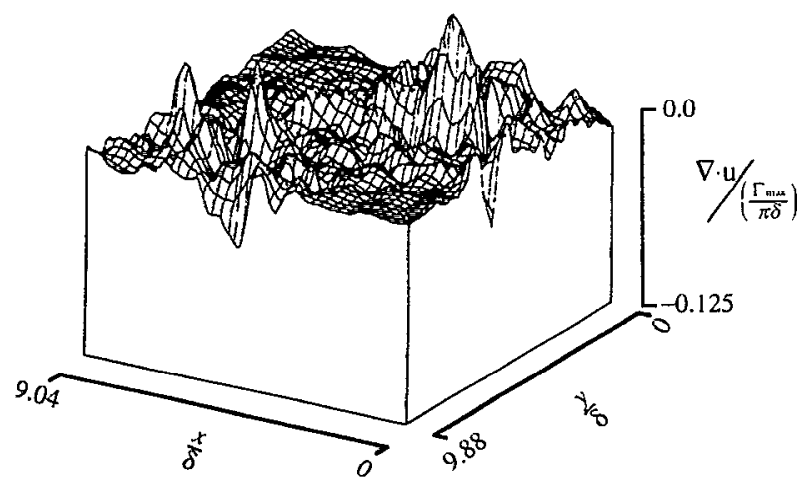

FIG. 28. The trace field obtained from the strain rate tensor field $\epsilon^{4}(x, t)$ in Fig. 27 for Case III in Sec. III C, shown on the same scale as the strain rate tensor components in Fig. 27 and demonstrating that continuity is essentially satisfied.

available. Owing to the relative magnitudes of the velocity gradient and scalar gradient length scales, $\lambda_{v}$ and $\lambda_{D}$, such fully resolved $S c \approx 1$ measurements would allow a much larger region in the velocity field to be examined than is presently the case for $\mathrm{Sc} \gg 1$ scalar measurements of the type in Ref. 43.

Current refinements of the technique are focusing on ways to accelerate the convergence, in part through methods mentioned in Sec. II, and on various numerical implementation issues aimed at velocity error minimization. In other work, we are applying the technique in experimental studies of the structure of the vorticity, strain rate, and pressure gradient fields in real turbulent flows, and on the influence of these dynamical fields on the fine structure of the scalar mixing process. Preliminary results suggest that this scalar imaging velocimetry technique provides a means for experimentally investigating the precise fine structure and dynamics of real turbulent flows at a level of detail previously conceivable only from large-scale direct numerical simulations (DNS) of the full Navier-Stokes equations.

\section{ACKNOWLEDGMENTS}

This work is supported, in part, by the Air Force Office of Scientific Research (AFOSR) under AFOSR Grants No. 91-2953 and No. 89-0541.

\section{APPENDIX: NUMERICAL IMPLEMENTATION}

Temporal evolution of the scalar fields involved representations of the fields at discrete time intervals $d t$. For Case I, these $\zeta(\mathbf{x}, t+d t)$ fields were created analytically, the field having been defined to include a (nondissipative) dependence on the given velocity field. In this way five fields were created, and the time differentiation was done using a fourth-order central difference. For Cases II and III, a second-order Runge-Kutta integration scheme, incorporating the specified velocities, gave the scalar field for two forward time steps, again ignoring dissipation. We treated the field from the first forward time step as the center plane $\zeta(x, t)$, by the notation of Sec. II. The time differentiation then used second-order central differences. The desire to lessen the influence of numerical errors in the advection motivated the decision to use only three planes in the temporal direction. All spatial differentiation was done using fourth-order central differences, with the exception of the edges of the scalar fields, where fourth-order one-sided schemes were used. All the scalar field values were specified as 8-bit numbers.

To obtain the zeroth iteration results $\mathbf{u}^{0}(\mathbf{x}, t)$, Eq. (6) was applied to all points in the data field that had a scalar gradient vector magnitude above a preselected threshold, rather than merely to those points at which the scalar gradient layers folded over onto themselves. It was found that the resulting $\mathbf{u}^{0}(\mathbf{x}, t)$ for each case showed excellent agreement with the known velocities over the entire field. This appears to confirm the arguments in Sec. II B that, at large Schmidt numbers, $\hat{e}_{\nabla \zeta}(\mathbf{x}, t)$ varies over sufficiently smaller lengthscales than does $\mathbf{u}(\mathbf{x}, t)$, so that $\nabla \mathbf{u} \cdot \hat{e}_{\nabla \zeta}$ in Eq. (4) is indeed sufficiently small in comparison with $u \cdot \nabla \hat{e}_{\nabla \zeta}$ virtually everywhere in the test fields. It was thus not necessary to identify regions where this approximation is good to obtain the initial result for $\mathbf{u}^{0}(\mathbf{x}, t)$, and for this reason we could simply interpolate on a regular $30 \times 30$ grid to obtain the zeroth solution over the entire field for both velocity components. The velocity vector for each point was determined by averaging the results of the initial application of Eq. (6) within a small region around that point. A bilinear interpolation was applied on the grid, followed by a cubic spline interpolation to yield the continuous and differentiable zeroth solution. The spatial derivatives were then minimally smoothed to remove the spottiness caused by the cubic spline interpolation, and were then used as the input to the first iterative step. The successive iterations were based on an alternative form of Eq. (7) in which the differences between two points $\mathbf{x}$ and $\mathbf{x}+d \mathbf{x}$ are replaced by sums, namely

$$
\begin{aligned}
u_{\|}(\mathbf{x}+d \mathbf{x})+u_{\|}(\mathbf{x})= & \mathbf{u}^{k} \cdot\left[\hat{e}_{\nabla \zeta}(\mathbf{x}+d \mathbf{x})+\hat{e}_{\nabla \zeta}(\mathbf{x})\right] \\
& +\left(\nabla \mathbf{u}^{k-1} \cdot d \mathbf{x}\right) \cdot \hat{e}_{\nabla \zeta}(\mathbf{x}+d \mathbf{x}),
\end{aligned}
$$

where $\mathbf{u}^{k}$ and $\nabla \mathbf{u}^{k-1}$ are the values at $\mathbf{x}$. This is still the linear system of Eq. (7), but this form improves the conditioning of the system for small $d \mathbf{x}$. The $\mathbf{u}^{k}(\mathbf{x}, t)$ component fields from Eq. (11) were processed before each subsequent iteration in the same manner as described above for the zeroth solution.

\footnotetext{
${ }^{1}$ A. K. Gupta, J. Laufer, and R. E. Kaplan, "Spatial structure in the viscous sublayer," J. Fluid Mech. 50, 493 (1971).

${ }^{2}$ R. F. Blackwelder and R. E. Kaplan, "On the wall structure of the turbulent boundary layer," J. Fluid Mech. 76, 89 (1976).

${ }^{3}$ F. K. Browand and P. D. Weidman, "Large scales in the developing mixing layer," J. Fluid Mech. 76, 127 (1976).

${ }^{4}$ A. K. M. F. Hussain and A. R. Clark, "On the coherent structure of the axisymmetric mixing layer: a flow visualization study," J. Fluid Mech. 104, 263 (1981).

${ }^{5}$ J. Tso, L. S. G. Kovasny, and A. K. M. F. Hussain, "Search for largescale coherent structures in the nearly self-preserving region of a turbulent axisymmetric jet," J. Fluids Eng. 103, 503 (1981).

${ }^{6}$ M. G. Mungal and P. E. Dimotakis, "Mixing and combustion with low heat release in a turbulent shear layer," J. Fluid Mech. 148, 349 (1984).
} 
${ }^{7} \mathbf{J}$. Tso and F. Hussain, "Organized motions in a fully developed turbulent axisymmetric jet," J. Fluid Mech. 203, 425 (1989).

${ }^{8} \mathrm{~A}$. Glezer and D. Coles, "An experimental study of a turbulent vortex ring," J. Fluid Mech. 211, 243 (1991).

${ }^{9}$ R. J. Adrian, "Particle-imaging techniques for experimental fluid mechanics," Annu. Rev. Fluid Mech. 23, 261 (1991).

${ }^{10} \mathrm{R}$. J. Adrian, "Multi-point optical measurements of simultaneous vectors in unsteady flow-a review," Int. J. Heat Fluid Flow 7, 127 (1986).

${ }^{11}$ W. Merzkirch, Flow Visualization (Academic, New York, 1987).

${ }^{12} \mathrm{~W}$. Lauterborn and A. Vogel, "Modern optical techniques in fluid mechanics," Annu. Rev. Fluid Mech. 12, 223 (1984).

${ }^{13}$ L. Hesselink, "Digital image processing in flow visualization," Annu. Rev. Fluid Mech. 20, 421 (1988).

${ }^{14}$ R. B. Miles and D. M. Nosenchuck, "Three-dimensional quantitative flow diagnostics," in Advances in Fluid Mechanics Measurements, Lecture Notes in Engineering, edited by M. Gad-el-Hak (Springer-Verlag, Berlin, 1989), Vol. 45.

${ }^{15}$ F. P. Chiang and G. T. Reid, Optics and Lasers in Engineering (Elsevier, New York, 1988), Vol. 9, pp. 161-325.

${ }^{16}$ M. Gad-el-Hak, Advances in Fluid Mechanics Measurements (SpringerVerlag, Berlin, 1989).

${ }^{17}$ T. D. Dudderar and P. G. Simpkins, "Laser speckle photography in a fluid medium," Nature 270, 45 (1977).

${ }^{18}$ D. B. Barker and M. E. Fourney, "Measuring fluid velocities with speckle patterns," Opt. Lett. 1, 135 (1977).

${ }^{19} \mathrm{R}$. Grousson and S. Mallick, "Study of flow pattern in a fluid by scattered laser light," Appl. Opt. 16, 2334 (1977).

${ }^{20} \mathrm{R}$. Meynart, "Instantaneous velocity field measurements in unsteady gas flow by speckle velocimetry," Appl. Opt. 22, 535 (1983).

${ }^{21}$ R. Erbeck and W. Merzkirch, "Speckle photographic measurement of turbulence in an air stream with fluctuating temperature," Exp. Fluids 6, 89 (1988).

${ }^{22}$ L. P. Bernal and J. T. Kwon, "Surface flow velocity field measurements by laser speckle photography," in Proceedings of the 4th International Symposium on Applications of Laser Techniques to Fluid Mechanics (Instituto Superior Técnico, Lisbon, 1988).

${ }^{23} \mathrm{~K}$. Oberste-Lehn and W. Merzkirch, "Speckle photographic measurement of turbulence properties in a premixed flame," in Proceedings of the 7th Symposium on Turbulent Shear Flows (Stanford U.P., Stanford, CA, 1989), Vol. 1, p. 26.5

${ }^{24} \mathrm{~J}$. Keller and W. Merzkirch, "Interaction of a normal shock wave with compressible turbulent flow," Exp. Fluids 8, 241 (1990).

${ }^{25}$ P. E. Dimotakis, F. D. Debussy, and M. M. Koochesfahani, "Particle strcak velocity field measurements in a two-dimensional mixing layer," Phys. Fluids 24, 995 (1981).

${ }^{26} \mathrm{~T}$. Utami and T. Ueno, "Visualization and picture processing of turbulent fiow," Exp. Fluids 2, 25 (1984).

${ }^{27} \mathrm{~T}$. Utami and T. Ueno, "Experimental study on the coherent structure of turbulent open-channel flow using visualization and picture processing," J. Fluid Mech. 174, 399 (1987).

${ }^{28} \mathrm{~J}$. C. Agüi and J. Jiménez, "On the performance of particle tracking," J. Fluid Mech. 185, 447 (1987).

${ }^{29}$ A. A. Adamcyzk and L. Rimai, "Two-dimensional particle tracking velocimetry (PTV): technique and image processing algorithms," Exp. Fluids 6, 373 (1988).

${ }^{30}$ R. J. Adrian, P. W. Offutt, C. C. Landreth, Z.-C. Liu, and T. J. Hanratty, "Studies of liquid turbulence using double-pulsed particle correlation," in Proceedings of the 5th Symposium on Applications of Laser Techniques to Fluid Mechanics (Instituto Superior Técnico, Lisbon, 1990), Vol. 1, p. 15.4.

${ }^{31}$ D. C. Bjorkquist, "Particle image velocimetry analysis system," Proceedings of the 5th Symposium on Applications of Laser Techniques to Fluid Mechanics (Instituto Superior Técnico, Lisbon, 1990), Vol. 1, p. 12.1 .

${ }^{32}$ Z.-C. Liu, C. C. Landreth, R. J. Adrian, and T. J. Hanratly, "High resolution measurement of turbulent structure in a channel with particle image velocimetry," Exp. Fluids 10, 301 (1991).

${ }^{33}$ C. E. Willert and M. Gharib, "Digital particle image velocimetry," Exp. Fluids 10, 181 (1991).

${ }^{34} \mathrm{~K}$. Nishino, N. Kasagi, and M. Hirata, "Three-dimensional particle tracking velocimetry based on automated digital image processing," J. Fluids Eng. 111, 384 (1989).

${ }^{35}$ D. Papantoniuo and H.-G. Maas, "Recent advances in 3-D particle tracking velocimetry," in Proceedings of the 5th Symposium on Applications of Laser Techniques to Fluid Mechanics (Instituto Superior Técnico, Lisbon, 1990), Vol. 1, p. 18.4.

${ }^{36} \mathrm{G}$. Haussmann and $W$. Lauterborn, "Determination of size and position of fast moving gas bubbles in liquids by digital 3-D image processing of hologram rcconstructions," Appl. Opt. 19, 3529 (1980).

${ }^{37}$ P. H. Malyak and B. J. Thompson, "Particle displacement and velocity measurement using holography," Opt. Eng. 23, 567 (1984).

${ }^{38} \mathrm{~J}$. Scherer and L. P. Bernal, "Resolution characteristics of holographic particle image velocimetry," AIAA Paper No. 92-0009, AIAA, Washington, DC, 1988.

${ }^{39}$ C. Hassa, P. H. Paul, and R. K. Hanson, "Laser induced fluorescence modulation techniques for velocity measurements in gas flows," Exp. Fluids 5, 240 (1987).

${ }^{40}$ R. E. Falco and C. C. Chu, "Measurement of two-dimensional fluid dynamics quantities using a photochromic grid tracing technique," in Photomechanics and Speckle Metrology (SPIE, Bellingham, WA, 1987), Vol. 84, p. 706.

${ }^{4} \mathrm{t}$ W. J. A. Dahm and K. A. Buch, "High-resolution three-dimensional $\left(256^{3}\right)$ spatiotemporal measurements of the conserved scalar field in turbulent shear flows," in Turbulent Shear Flows (Springer-Verlag, Berlin, 1991), Vol. 7, pp. 17-26.

${ }^{42}$ W. J. A. Dahm, K. B. Southerland, and K. A. Buch, "Four-dimensional laser induced fluorescence measurements of conserved scalar mixing in turbulent flows," in Proceedings of the 5th International Symposium on Applications of Laser Techniques to Fluid Mechanics (Instituto Superior Técnico, Lisbon, 1990), Vol. 1, pp. 1.1-1.6; Applications of Laser Techniques to Fluid Mechanics (Springer-Verlag, Berlin, 1991).

${ }^{43}$ W. J. A. Dahm, K. B. Southerland, and K. A. Buch, "Direct, highresolution, four-dimensional measurements of the fine scale structure of Sc $\gg 1$ molecular mixing in turbulent flows," Phys. Fluids A 3, 1115 (1991).

${ }^{44}$ K. B. Southerland, J. R. Porter, W. J. A. Dahm, and K. A. Buch, "An experimental study of the molecular mixing process in an axisymmetric laminar vortex ring," Phys. Fluids A 3, 1385 (1991). 